\section{Kinetic target-guided synthesis in drug discovery and chemical biology: a comprehensive facts and figures survey}

For the last 15 years, kinetic target-guided syntheses, including in situ click chemistry, have been used as alternative methods to find ligands to therapeutically relevant proteins. In this review, a comprehensive survey of biological targets used in kinetic target-guided synthesis covers historical and recent examples. The chemical reactions employed and practical aspects, including controls, library sizes and product detection, are presented. A particular focus is on the reagents and warhead selection and design with a critical overview of the challenges encountered. As protein supply remains a key success factor, it appears that increased efforts should be taken toward miniaturization in order to expand the scope of this strategy and qualify it as a fully fledged drug discovery tool.

First draft submitted: 10 December 2015 Accepted for publication: 11 January 2016 Published online: 12 February 2016

Target-guided syntheses are strategies used for the last 20 years to discover ligands that are assembled 'by' the protein itself from biocompatible-reactive reagents. Only a few members of the virtual combinatorial library of products are actually produced and detected. Two main proteintemplated strategies have emerged: in situ dynamic combinatorial chemistry (DCC) and kinetic target-guided synthesis (KTGS) (Figure 1). In situ DCC assembles ligands via a reversible process (Figure 1A). The presence of the biological target shifts the thermodynamic equilibrium between products resulting from a reversible reaction (i.e., imine formation) [1]. The dynamic combinatorial libraries are also called 'in vitro virtual combinatorial libraries' [2]. Using this strategy, inhibitors of neuraminidase from influenza virus have been discovered [2].

On the other hand, in KTGS $[3,4]$ (Figure 1B), the biological target accelerates an irreversible reaction between a pair of reagents by stabilizing a productive configuration of the ternary complex. If the prod- uct is structurally similar to the transition state, its affinity for the protein is significantly improved compared with the affinity of each reagent. The most used KTGS approach is the in situ click chemistry in the case of copper-free cycloaddition of azides and alkynes. It is an extension of the click reaction concept. It was pioneered by Sharpless and colleagues and can be advantageously used for the discovery of hits in medicinal chemistry [5].

The term 'click' refers to reactions that are selective, high yielding and that display good kinetics. A widely used reaction is the 1,3-dipolar cycloaddition for which a $\mathrm{Cu}$ catalyzed version has been developed [6,7]. When the reaction conditions are biocompatible and the reagents are biorthogonal [8], the click reactions can be used to label biomolecules, or in cells. Also, they allow parallel microplate-scale synthesis of ligands followed by biological screening without purification ('in situ screening') [9,10].

Both DCC and KTGS are of huge potential in drug-discovery though they remain unconventional and relatively unexplored.
Damien Bosc' ${ }^{1}$, Jouda Jakhlal', Benoit Deprez ${ }^{1}$ \& Rebecca Deprez-Poulain $*, 1$

IInserm, Institut Pasteur de Lille, U1177 Drugs \& Molecules for Living Systems, Université de Lille, F-59000 Lille, France *Author for correspondence: rebecca.deprez@univ-lille2.fr 


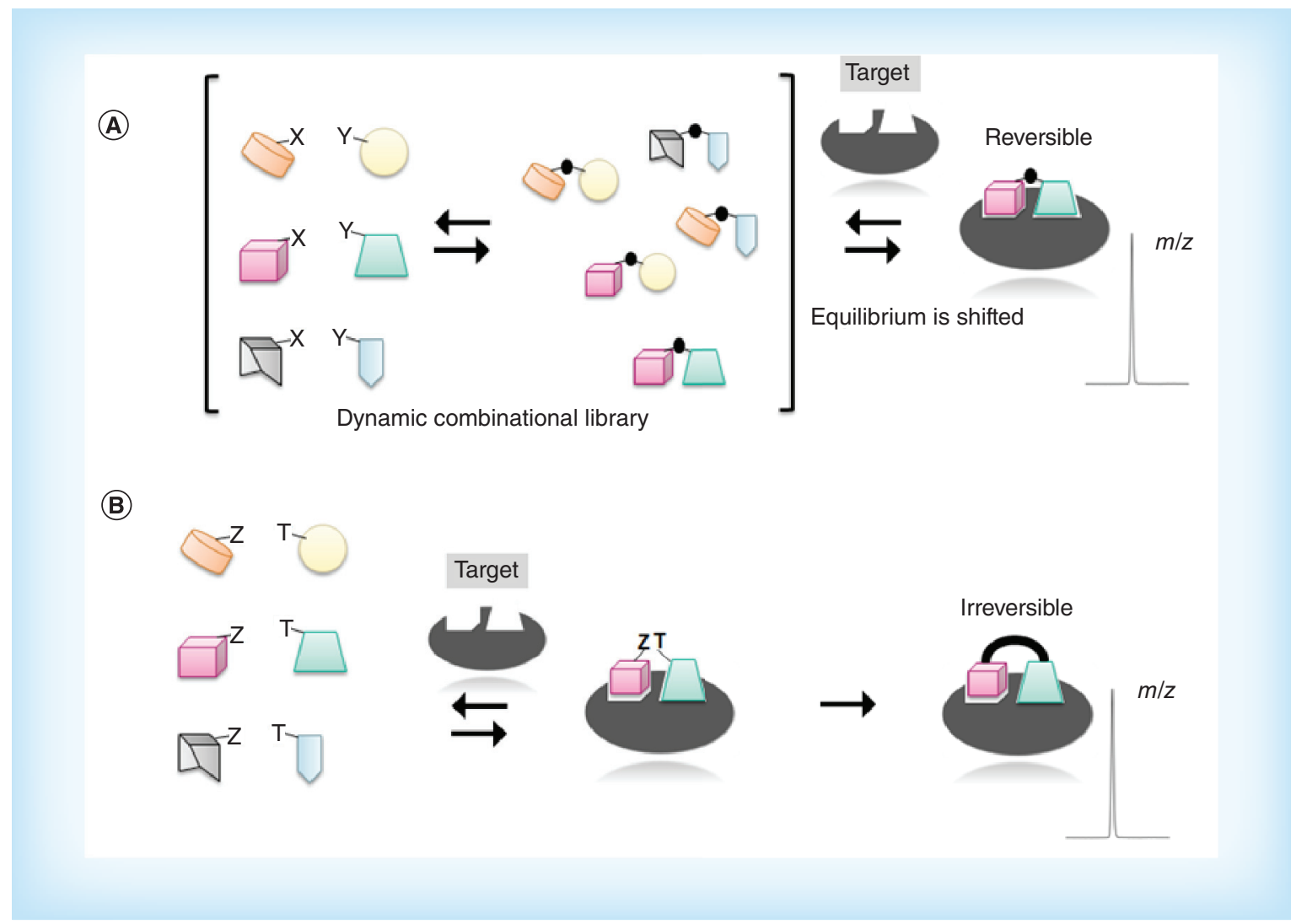

Figure 1. Principles of in situ dynamic combinatorial chemistry and kinetic target-guided synthesis. (A) In situ dynamic combinatorial chemistry, the target displaces the equilibrium of the library members formed by a reversible reaction between reagents $X$ and $Y$ toward the library member(s) that bind efficiently to the biological target. Reversible reactions are, for example, imine formation and boronic ester formation. (B) In kinetic targetguided synthesis, the target stabilizes a pair of reagents in reacting relative configurations and therefore accelerates the irreversible formation of the product. Irreversible reactions are, for example, click chemistry between an azide and an alkyne. In both cases, detection of the ligand is performed by MS techniques.

Recently, the fragment-based drug discovery approach has been successful in providing leads and drugs, like vemurafenib a B-Raf inhibitor [11] and has attracted a large community of scientists [12]. Fragment-based drug discovery makes use of small molecules (fragments) and x-ray crystallography or nuclear magnetic resonance (NMR) spectroscopy screening techniques. Optimization of a fragment into a lead can be performed by: linking several fragments; growing and evolving the fragment and/or, in situ fragment linking (i.e., using DCC and KTGS) [13].

KTGS starts with the selective binding of reagents to specific pockets in the protein targeted-binding site. Then, if two complementary reagents are in the suitable proximity and relative configuration, the irreversible reaction occurs and allows the formation of an energetically more favorable new complex with the protein. KTGS does not require the prior synthesis and purification of library compounds nor the screening of each final product. It is thus more efficient, or at least complementary to traditional combinatorial chemistry (parallel synthesis) or fragment-based drug design.

\section{Biological applications of KTGS}

General analysis of the therapeutic scope of published examples

Historically, KTGS has been applied to the discovery of potent inhibitors of acetylcholine esterase (AChE) by Sharpless's team [5]. Since then and until recently, KTGS has been mainly applied to the discovery of ligands of enzymes ( $72 \%$ of targets, Figure $2 \mathrm{~A})$. Nevertheless, some other target classes have recently been tackled. Discovery of ligands of a neurotransmitter ligand-gated ion channel (ACh-binding protein) [14] and, of a receptor of the TetR family (EthR) [15] implicated in the resistance to ethionamide $(\mathrm{ETH})$ bioactivation by Mycobacterium tuberculosis, has been disclosed.

Regarding enzymes, five of the six different classes of enzymes have been used in KTGS, enlightening the large scope of these syntheses (Figure 2B): hydrolases, 
ligases, transferases, lyases (carbonic anhydrase [16]) and oxido-reductases. In the large class of hydrolases, the type of enzymes and mechanisms are also diverse, ranging from esterases [5], proteases [17,18], glucosidases [19] or deacetylases [20]. Recent drug-discovery relevant targets, like kinases, have also been explored (BcR-Abl [21] or NAD-kinase of bacteria [22]).

The study of protein-protein interactions is a rising field in drug discovery [23,24]. KTGS has also been applied to this field leading to the discovery of inhibitors of $\mathrm{Bcl}-\mathrm{XL} / \mathrm{BAX}$ interactions $[25,26]$ and protein 14-3-3 $\zeta$ [27]. Interestingly, these examples involve large cavities more suitable for reagent binding than flat surfaces frequently found in protein-protein complexes.

A total of 29 different KTGS experiments have been disclosed on 14 different biological targets. Eleven of these experiments were performed on AChE from various animal species, the rest being performed on diverse human and nonhuman targets.

As depicted in Figure 2D, proteins used for KTGS are involved in very different therapeutic areas like cancer, ophthalmology, metabolic diseases, CNS disorders. In particular, a higher proportion of proteins are studied for their implication in infectious diseases (AIDS, tuberculosis and other bacterial infections). This may reflect the fact that almost all reports of KTGS arise from academic groups.

\section{Biological characterization from in vitro to in vivo}

Acetylcholine (ACh) is a neurotransmitter that binds nicotinic receptors (nAChR) after its release in the synapse, and which is inactivated by the serine AChE. $\mathrm{AChE}$ is a target for Alzheimer's disease drugs such as galantamine (Reminyl ${ }^{\mathrm{TM}}$ ) and donezepil (Aricept ${ }^{\mathrm{TM}}$ ), glaucoma drug physostigmine (Isopto ${ }^{\mathrm{TM}}$ ) and myasthenia gravis neostigmine (Prostigmin ${ }^{\mathrm{TM}}$ ) [28]. AChE is also present in plasma where it is involved, like other esterases, in the metabolism of ester prodrugs [29]. Sharpless and Renard discovered several closely related AChE inhibitors by KTGS (Figure 3A). The first series consisted in inhibitors displaying tacrine and phenanthridinium pharmacophores to target both the catalytic and the peripheral site and identified syn-TZ2PA6 and syn-TZ2PA5 as femtomolar inhibitors of the enzyme $[5,30]$. Close equipotent analogs were discovered subsequently based on phenyltetrahydroisoquinoline in place of phenanthridinium [31] TZ2PIQ-A5 and TZ2PIQ-A6. Inspired by this work, a series of huprinederived inhibitors of $\mathrm{AChE}$ was identified using either Huisgen reaction [32] (9-HUPZ4PIG-A2) or thioMichael addition (H3-P2) [33]. Nevertheless, though very potent, none of the above $\mathrm{AChE}$ inhibitors were progressed to cellular or more complex biochemical assays.

$\mathrm{nAChR}$ is a ligand-gated ion channel, for which agonists are in development with applications in schizophrenia, nicotine addiction and Alzheimer's disease $[34,35]$. The acetylcholine-binding protein (AChBP) is a soluble protein from gastropods and polychaetes. It displays a structure that is homologous to the extracellular domain of nAChR, providing the same binding sites for $\mathrm{ACh}$ at the subunits interfaces. Grimster et al. disclosed a KTGS that allowed the identification of compound 18 (Figure 3C), a low nanomolar triazole inhibitor [14].

Carbonic anhydrase-II (CA-II) is a protein that catalyzes the interconversion between $\mathrm{CO}_{2}$ and $\mathrm{HCO}_{3}^{-}$. It is thus implicated in the regulation of blood $\mathrm{pH}$, renal reabsorption of $\mathrm{NaCl}$ and bicarbonate in the proximal tubule and in secretion of aqueous humor in the ciliary body of the eye. The class of sulfonamides that inhibit CA-II by interacting with the catalytic zinc has provided several drugs, such as dorzolamide (Trusopt ${ }^{\mathrm{TM}}$ ) for glaucoma [36] and acetazolamide (Diamox ${ }^{\mathrm{TM}}$ ) as a diuretic [37].

Huc published the first CA-II inhibitor discovered by KTGS [38] (Compound 3e; Figure 3B) while Kolb et al. published other inhibitors with improved potency [16] (Compound 5; Figure 3B). The same strategy of KTGS was used to develop radiopharmaceutical chemical probes for positron emission topography (PET) [39]. Compound $\left[{ }^{18} \mathrm{~F}\right]-3 \mathrm{~b}$ (Figure 3B) was used as a PET probe to target CA-II in mice. The distribution of the tracer in blood, lungs, heart and kidney is well correlated with known distribution of CA-II. In particular, the distribution in blood was confirmed to be CA-II dependent using displacement by a cold inhibitor of CA-II. The hepatic metabolism of the probe was also explored. This work is a proof-of-concept of the use of KTGS for imaging purposes in drug discovery. In the same line, a templated reaction allowed the identification of a fluorinated compound $\mathbf{6}$ (Figure 3C) [40] as a precursor for a PET probe for human cyclooxygenase-2 (bCOX-2), a target for nonsteroidal anti-inflammatory drugs (celecoxib; Celebrex ${ }^{\mathrm{TM}}$ ).

Several compounds have been developed for infectious diseases, HIV-1 protease is a target for the treatment of AIDS. Numerous inhibitors are available on the market and are used in cocktails with other pharmacological classes to tackle the virus resistance. Sharpless and colleagues discovered an inhibitor of HIV protease inspired by indinavir $\left(\mathrm{IC}_{50}=6 \mathrm{nM}\right.$; anti-3; Figure 3C) [17].

Chitinases are biological targets for antifungals, antiparasitics and antibacterials. Omura et al. disclosed an inhibitor with a low nanomolar activity of different 


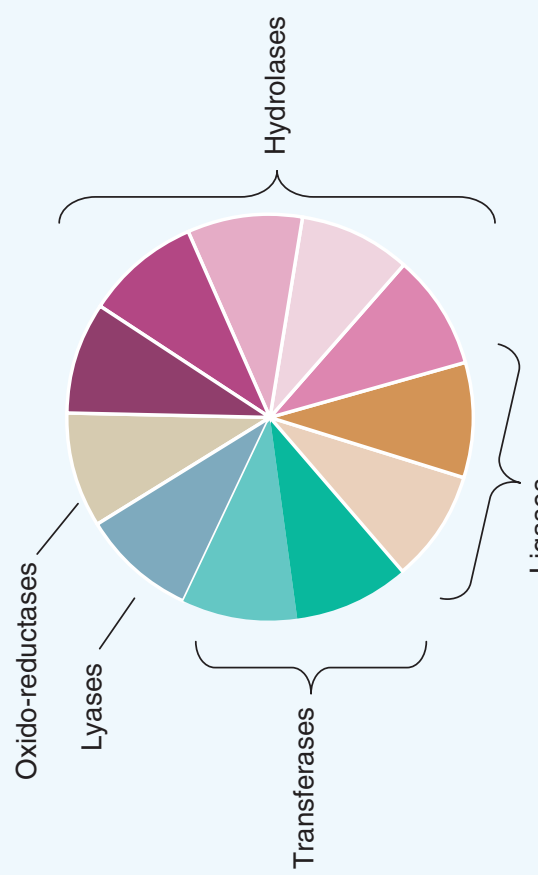

(9)

(4)
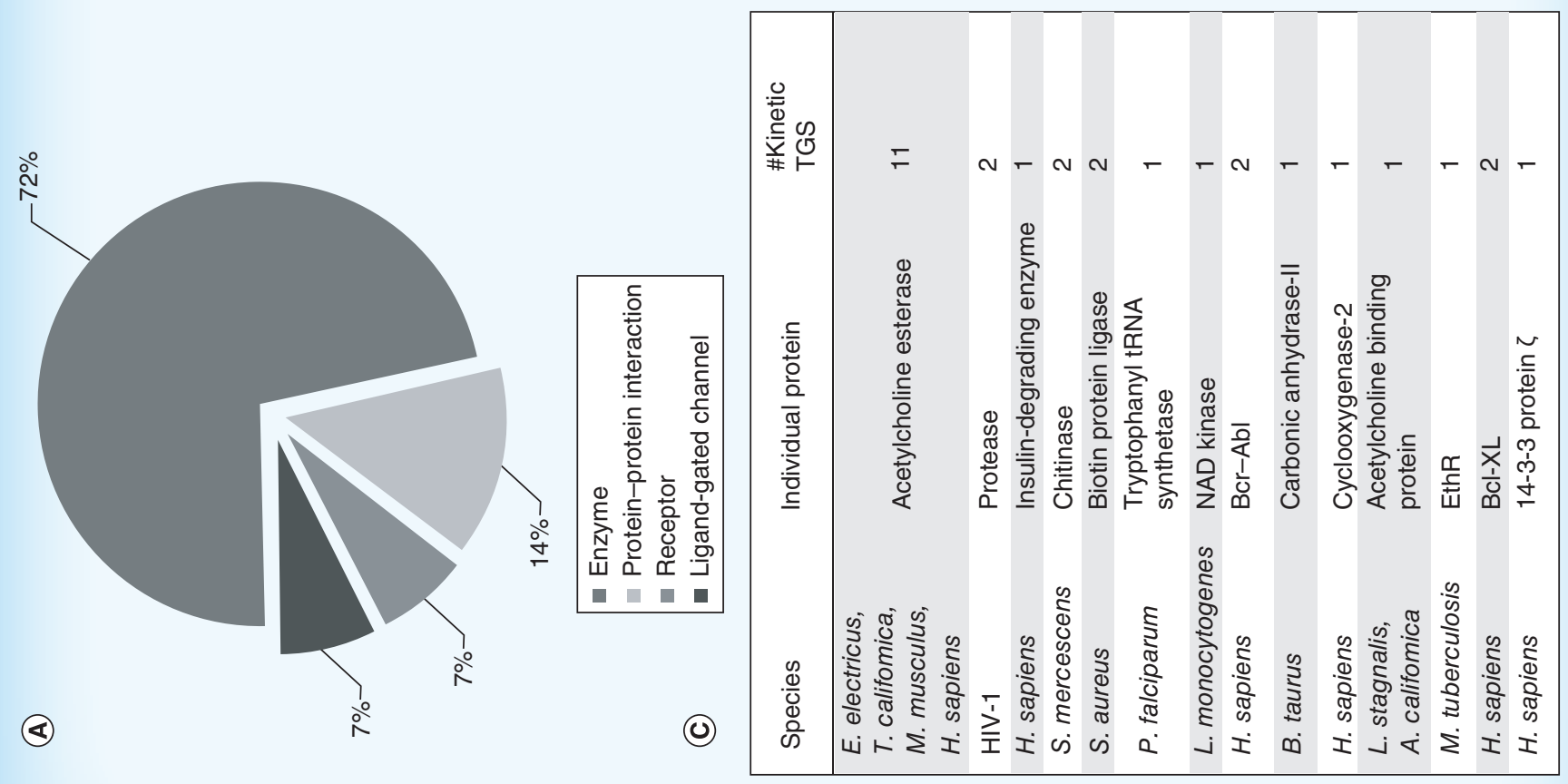
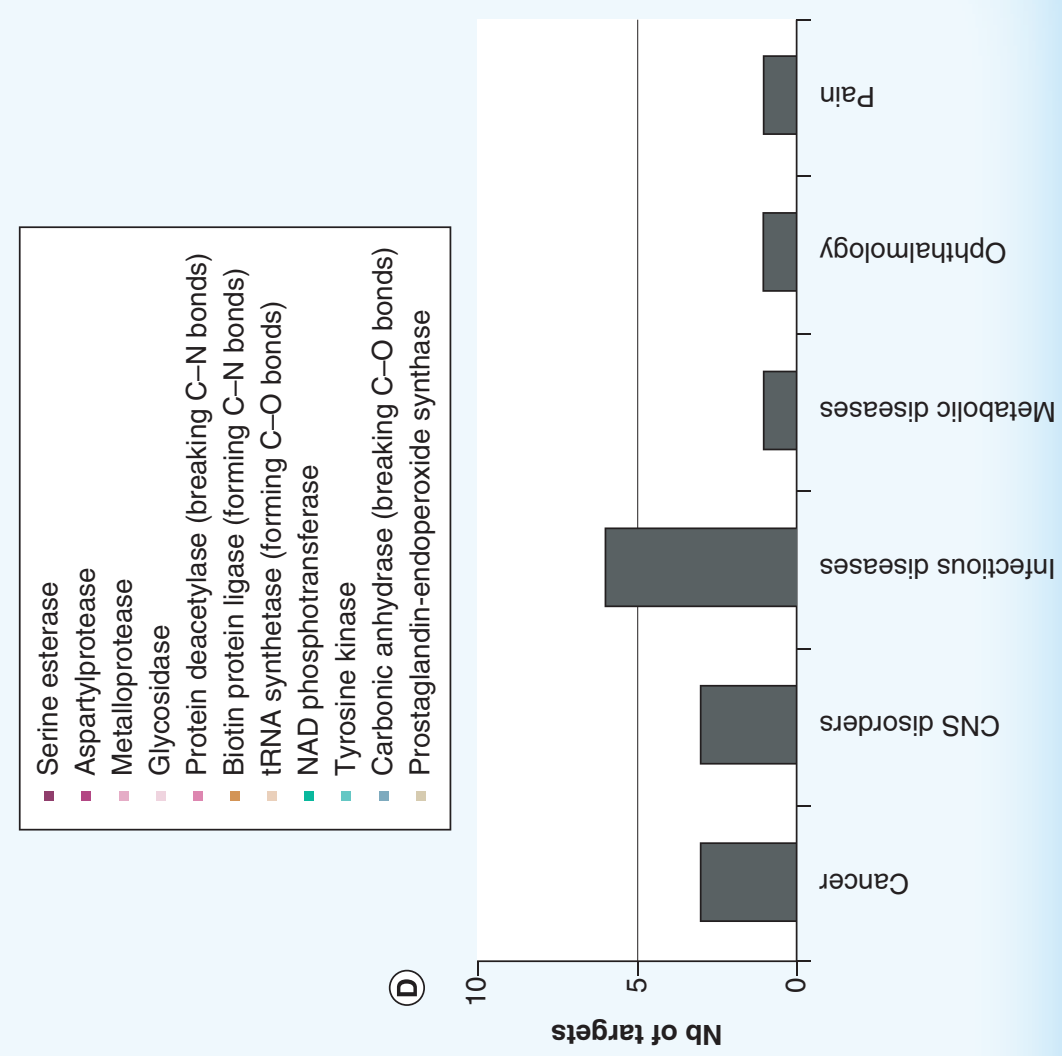

(a) 으

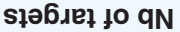


Figure 2. Types of biological targets, number of experiments and therapeutic applications of kinetic target-guided synthesis in the literature (see facing page). (A) Distribution of proteins by class. (B) Distribution of enzymes by subclass. (C) Individual proteins (name and species) used in kinetic target-guided synthesis and the number of experiments (\#) described. (D) Potential therapeutic applications of ligands discovered through kinetic target-guided synthesis.

isoforms of chitinases of Serratia marcescens (SmChi), a Gram-negative bacteria (syn-7; Figure 3C) [19].

Gelin et al. described the screening of adenosine derivatives and discovered that a KTGS templated by the NAD kinase from Listeria monocytogenes (LmNADK1) occurred. The resulting dinucleoside (15; Figure 3C) inhibits bacterial NAD kinases from L. monocytogenes and Staphylococcus aureus and blocked bacterial growth at concentrations similar to vancomycin. Moreover, the best compound did not show any cytotoxicity. This discovery is important since it tackles an enzyme that is not targeted by current antibiotics [22].

Tuberculosis is an infectious disease caused by the bacteria Mycobacterium tuberculosis. Ethionamide (ETH), a second-line antibiotic, is a prodrug that is activated in the bacteria by EthA, a monooxygenase. The active form of ETH (adduct with NAD) inhibits InhA, a protein involved in the biosynthesis of mycolic acid an essential component of the bacteria wall. EthA is under control of a transcriptional repressor EthR. Thus, inhibiting EthR boosts sensitivity of M. tuberculosis to ETH [41]. Willand et al. disclosed a EthR-templated KTGS that identified BDM14950 ( $\mathrm{IC}_{50}=944 \mathrm{nM}$; Figure 3C) paving the way to new inhibitors with new binding mode [15].

Finally, biotin protein ligase (BPL) is another potential bacterial target that is involved in the synthesis of biotinyl-5'-AMP, a key intermediate essential for the biotinylation of enzymes of the fatty acid biosynthesis. Tieu et al. described a KTGS of a known triazole using S. aureus BPL (triazole 8; Figure 3C) [42].

In the cancer field, two different recent target families were explored: kinases and protein-protein interactions. BcR-Abl is an essential protein inhibited by imatinib (Gleevec $\left.{ }^{\mathrm{TM}}\right)$, the first-line treatment for chronic myeloid leukemia. BcR-Abl can template the synthesis of its own inhibitors, like FA030 (Figure 3C; $\mathrm{IC}_{50}=0.9 \mu \mathrm{M}$ ), a previously known inhibitor for which the antiproliferative effect in cells had been shown $\left(\mathrm{IC}_{50}=0.89 \mu \mathrm{M}\right.$ on the $\mathrm{K}-562$ cell line) [21]. Manetsch reported a KTGS templated by Bcl-XL, an antiapoptotic protein of the $\mathrm{Bcl}-2$ family implicated in numerous protein-protein interactions with members of the Bax family [43]. SZ7TA2 (Figure 3C) was identified as the best compound and has been shown to modulate $\mathrm{Bcl}-\mathrm{XL} / \mathrm{Bak}_{-} \mathrm{BH}_{3}$ interactions [25].

In the field of metabolic diseases, insulin-degrading enzyme (IDE), a metalloprotease responsible for the inactivation of numerous bioactive peptides among which insulin and amyloid- $\beta$, has been proposed as a putative drug target in diabetes. Thanks to KTGS, the first catalytic site inhibitor of IDE (BDM44768; Figure 3C) to be tested in vivo has been developed [18]. The compound was characterized for its binding to the target by cocrystallization with the target along with NMR studies. Activity was assessed in cells and pharmacokinetic properties were measured in vitro and in vivo. Acute treatment of mice with BDM44768 increases insulin signaling and surprisingly impairs glucose tolerance in an IDE-dependent manner. This compound is the first KTGS compound to be tested in vivo for pharmacodynamics [18].

Figure 3D shows that only 35\% of compounds display a molecular weight $(\mathrm{MW})<500 \mathrm{~g} / \mathrm{mol}$. This may be a serious issue for the optimization of these compounds. Nevertheless, it should be noted that some of the published examples included in our statistics were previously optimized ligands produced to prove that KTGS could be used with the target of interest.

We have calculated both ligand efficiency (LE) and lipophilic ligand efficiency ([LLE]; Figure 3D). LE is the measurement of the binding energy of a ligand to its target per heavy atom (i.e., its size). It is calculated by the equation: $\mathrm{LE}=1.4\left(-\log \mathrm{IC}_{50}\right) / \mathrm{N}$. The reference value is 0.5 (compound with a $\mathrm{pIC}_{50}$ of 8 and 25 heavy atoms). LLE is the measurement of the activity of a ligand per unit of lipophilicity. It is calculated by the equation: lipophilic efficiency $=\mathrm{pIC}_{50}-\operatorname{cog} \mathrm{P}($ or $\log \mathrm{D})$. The reference value is 6 (compound with a $\mathrm{pIC}_{50}$ of 8 and a $\operatorname{cog} \mathrm{P}$ of 2). Compounds arising from KTGS are not expected to bear large excess of useless atoms or structural groups as they fully derive from binding reagents. Noteworthy, the smaller compounds have also the higher LE (above 0.3) and lipophilic efficiency (above 4). Among the most interesting compounds with this respect are $\mathbf{3 b}$ (inhibitor of CA-II), 18 (ligand of AChBP) and BDM44768 (inhibitor of IDE). KTGS thus proves to be suitable to identify leads with optimized physicochemical and pharmacological properties.

\section{Click synthetic strategies}

Several chemical reactions possess 'click reaction' characteristics that make them suitable for KTGS [44,45]. Figure 4 summarizes the main 'click reactions' disclosed so far. The first criteria for a click reaction is a high yield with a good conversion into expected product, in other words, thermodynamically favored, to avoid if possible complex purification steps. If any by-products are 
(A)

R-<smiles>c1ccc2c(NCCn3ccnn3)c3c(nc2c1)CCCC3</smiles>

R- :<smiles></smiles>

$\mathrm{n}=5$ : syn-TZ2PA5 ee/AChE K $K_{d}=100 \mathrm{fm}$ $m A C h E K_{d}=2300 \mathrm{fm}$

$\mathrm{n}=6:$ syn-TZ2PA6 ee/AChE K $K_{d}=99 \mathrm{fM}$ mAChE $K_{d}=410 \mathrm{fM}$<smiles>[R5][C@]1([2H])c2cc(OC)c(OC)cc2CCN1CCC</smiles>

(B)<smiles>NS(=O)(=O)c1ccc(CSCc2cccc(C(=O)O)c2)cc1</smiles>

$3 e$

Carbonic anhydrase II Bovine $\mathrm{K}_{\mathrm{i}}=59 \mathrm{nM}$

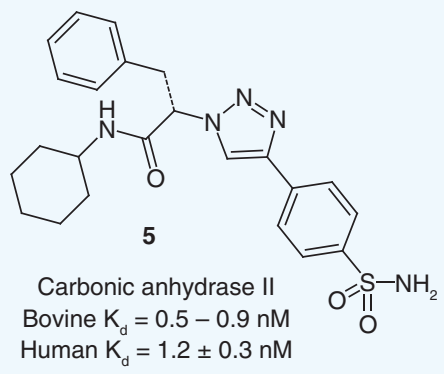

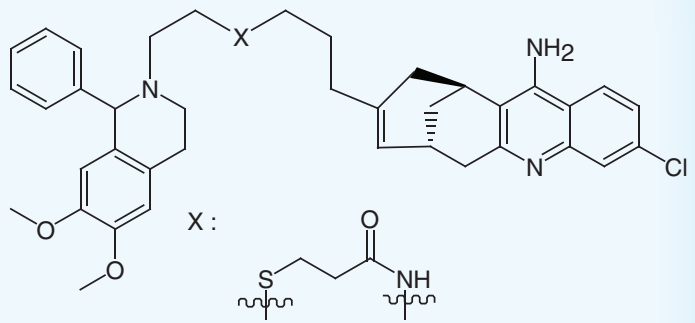

( \pm )-H3-P2

mAChE IC ${ }_{50}=0.9 \pm 0.1 \mathrm{nM}$

$\mathrm{X}$ :<smiles>CCn1cc(CO)nn1</smiles>

( \pm -9-HUPZ4PIQ-A2

mAchE IC ${ }_{50}=0.4 \pm 0.1 \mathrm{nM}$ rhAChE IC I0 $_{50}=0.6 \pm 0.1 \mathrm{nM}$

(C)

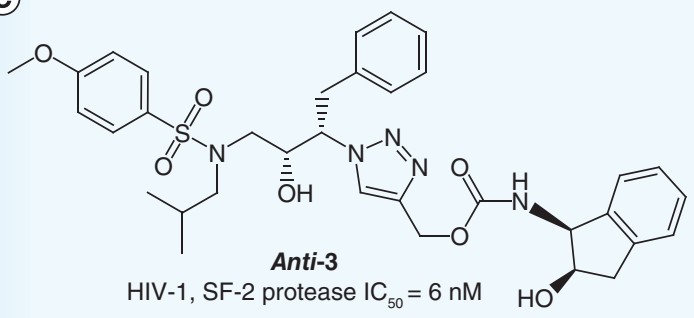

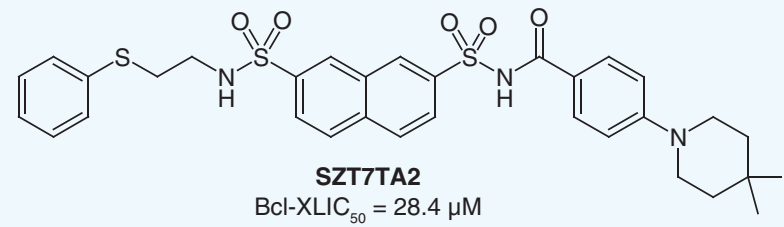<smiles>CCCc1cn(CCCCn2cnc3c(N)ncnc32)nn1</smiles><smiles>CCC[C@H]1SC[C@@H]2NC(=O)N[C@@H]21</smiles>

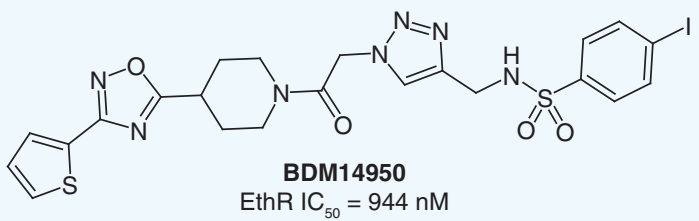<smiles>CNC(=O)NC(=N)NCCC[C@H](NC(=O)Cc1ccccc1)C(=O)N(Cc1ccccc1)Cc1ccccc1</smiles><smiles>CCn1nncc1CO/N=C/c1ccnc2ccccc12</smiles>

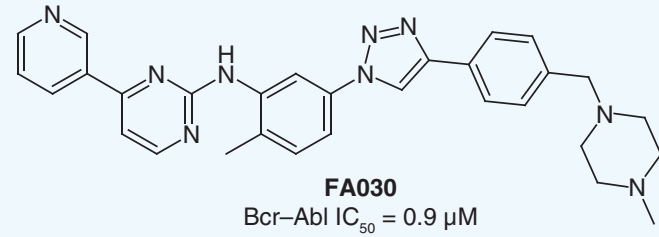


<smiles>NC[C@H]1O[C@H](n2c(SCC(=O)NC[C@@H]3O[C@H](n4c(Br)nc5c(N)ncnc54)[C@H](O)[C@H]3O)nc3c(N)ncnc32)[C@H](O)[C@H]1O</smiles>

LmNADK K $K_{\mathrm{i}}=25 \mu \mathrm{M}$

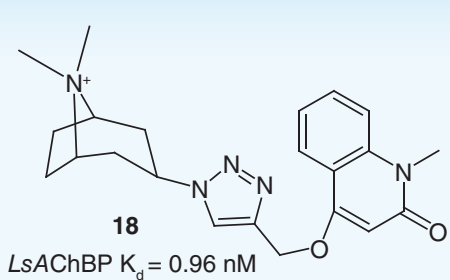

LsAChBP K $=0.96 \mathrm{nM}$

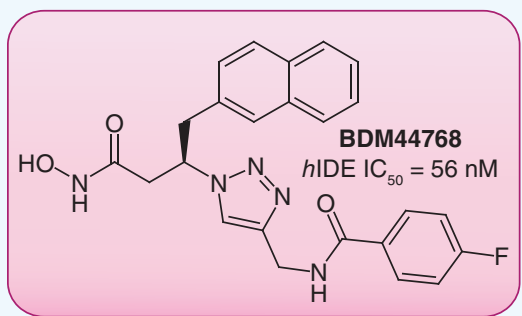

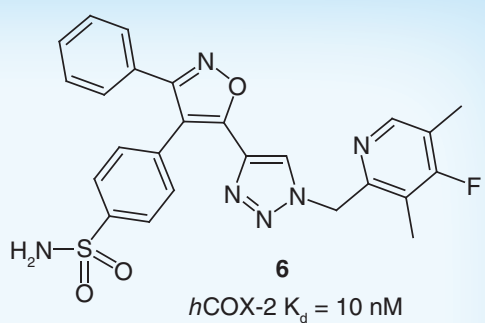

$h \operatorname{COX}-2 \mathrm{~K}_{\mathrm{d}}=10 \mathrm{nM}$

(D)

\begin{tabular}{|lllllllll|}
\hline Cpd & cLogP & PSA $\left(\AA^{2}\right)$ & HBA & HBD & $\begin{array}{l}\text { MW } \\
(\mathbf{g} / \mathbf{m o l})\end{array}$ & $\begin{array}{l}\text { \# Heavy } \\
\text { atoms }\end{array}$ & LE & LLE \\
\hline syn-TZ2PA5 & 8.8 & 111.6 & 6 & 3 & 661.9 & 50 & 0.28 & 1.2 \\
\hline syn-TZ2PIQ-A5 & 8.0 & 77.3 & 7 & 1 & 630.8 & 47 & 0.31 & 2.4 \\
H3-P2 & 7.6 & 115.0 & 7 & 2 & 711.4 & 50 & 0.25 & 1.5 \\
\hline 9-HUPZ4PIQ-A2 & 9.0 & 116.6 & 8 & 1 & 721.4 & 51 & 0.26 & 0.4 \\
\hline 3e & 2.3 & 131.1 & 5 & 2 & $\mathbf{3 3 7 . 4}$ & 22 & $\mathbf{0 . 4 6}$ & $\mathbf{4 . 9}$ \\
\hline $\mathbf{5}$ & 3.6 & 128.4 & 5 & 2 & $\mathbf{4 5 3 . 6}$ & 32 & $\mathbf{0 . 4 1}$ & $\mathbf{5 . 7}$ \\
3b & 1.9 & 141.2 & 6 & 2 & $\mathbf{4 3 2 . 5}$ & 30 & $\mathbf{0 . 4 3}$ & $\mathbf{7 . 4}$ \\
anti-3 & 4.5 & 164.5 & 9 & 3 & 663.8 & 47 & 0.24 & 3.7 \\
syn-7 & 3.0 & 191.6 & 9 & 5 & 703.8 & 52 & 0.21 & $\mathbf{4 . 6}$ \\
\hline BDM14950 & 2.3 & 172.7 & 7 & 1 & 639.5 & 36 & 0.23 & 3.7 \\
\hline 18 & 0.4 & 60.3 & 4 & 0 & 394.5 & 29 & $\mathbf{0 . 4 4}$ & $\mathbf{8 . 6}$ \\
\hline 15 & -2.6 & 319.0 & 17 & 8 & 683.5 & 43 & 0.15 & $\mathbf{7 . 2}$ \\
\hline FA030 & 4.3 & 107.1 & 9 & 2 & 521.6 & 39 & 0.22 & 1.7 \\
\hline SZT7tA2 & 5.7 & 154.7 & 7 & 2 & 637.8 & 43 & 0.15 & -1.2 \\
\hline Triazole 8 & 1.9 & 166.8 & 8 & 3 & $\mathbf{4 7 0 . 6}$ & 33 & 0.26 & $\mathbf{4 . 3}$ \\
\hline 6 & 4.7 & 138.2 & 6 & 1 & 504.5 & 36 & $\mathbf{0 . 3 1}$ & 3.3 \\
BDM44768 & 3.2 & 109.1 & 5 & 3 & $\mathbf{4 4 7 . 5}$ & 33 & $\mathbf{0 . 3 1}$ & $\mathbf{4 . 1}$ \\
\hline
\end{tabular}

Figure 3. Compounds identified via kinetic target-guided synthesis (see facing page for A \& B). (A) Inhibitors of AChE, (B) inhibitors of CA-II, and (C) inhibitors of others proteins. In color are depicted compounds used in vivo: in green, a ligand used for imaging purposes; and in magenta, the first compound tested for in vivo pharmacokinetics and pharmacodynamics; (D) calculated parameters (PipelinePilot ${ }^{\mathrm{TM}}$ ), in bold MW <500; LE $>0.3$ and LipE $>4$.

AChE: Acetylcholine esterase; Bcl-XL: B-cell lymphoma-extra large; Chi: Chitinase; COX-2: Cyclooxygenase-2; Cpd: Compound; EthR: Transcriptional repressor of ethionamide; HBA Hydrogen-bond acceptor; HBD: Hydrogen-bond donor; IDE: Insulin-degrading enzyme; LE: Ligand efficiency; LipE: Lipophilic efficiency; MW: Molecular weight; PSA: Polar surface area. 
formed, they should be easily removed and should not interact with the target. The click reaction should also be biocompatible or bioorthogonal (i.e., fully compatible with biological systems [proteins and cells]: chemically selective, inert, nontoxic, occurring in water, at physiological $\mathrm{pH}$ and temperature) and proceed in physiological conditions [46]. The transition state should not be sterically and electronically too different from the reagents and the product. This is well obeyed in cycloaddition reactions. Any important conformational change in the reaction path is likely to prevent KTGS.

The Huisgen 1,3-dipolar cycloaddition between 1,3-dipole azides and dipolarophile alkynes gives 1,2,3-triazoles. Both azides and alkynes are bioorthogonal reagents. A proof-of-concept was disclosed by Mock et al. with cucurbituril, a synthetic receptor $[47,48]$. NMR revealed that the ammonium side chain of reagent underwent interactions with cucurbituril urea subunits, allowing good positioning of the azide and alkyne thus favoring formation of the triazole. Also, this reaction was named 'in situ click chemistry' when used for KTGS and is the first reaction ever used for this purpose. An interesting advantage of the Huisgen reaction is its lack of regioselectivity producing both syn- and anti-triazoles [49], thus enhancing the chemical diversity of potential ligands without expanding the number of reagents nor the quantity of target required. Nevertheless, azide design and synthesis should take into consideration the specific hazard linked to their reactive properties. Indeed, azides and their salts are sensitive toward shock and friction. Thus, adequate safety precautions should be used for the synthesis, purification, isolation and handling of such compounds and the waste derived from their use.

One other reaction suitable for KTGS is the sulfoclick reaction $[25,26]$. Williams et al. reported that electron-deficient azides (sulfonyl azides) or electron-rich azides (alkyl/aryl azides) react with thio acids to give acylsulfonamides [50,51].

The direct amidation between amine and carboxylic acid group has also been found suitable for

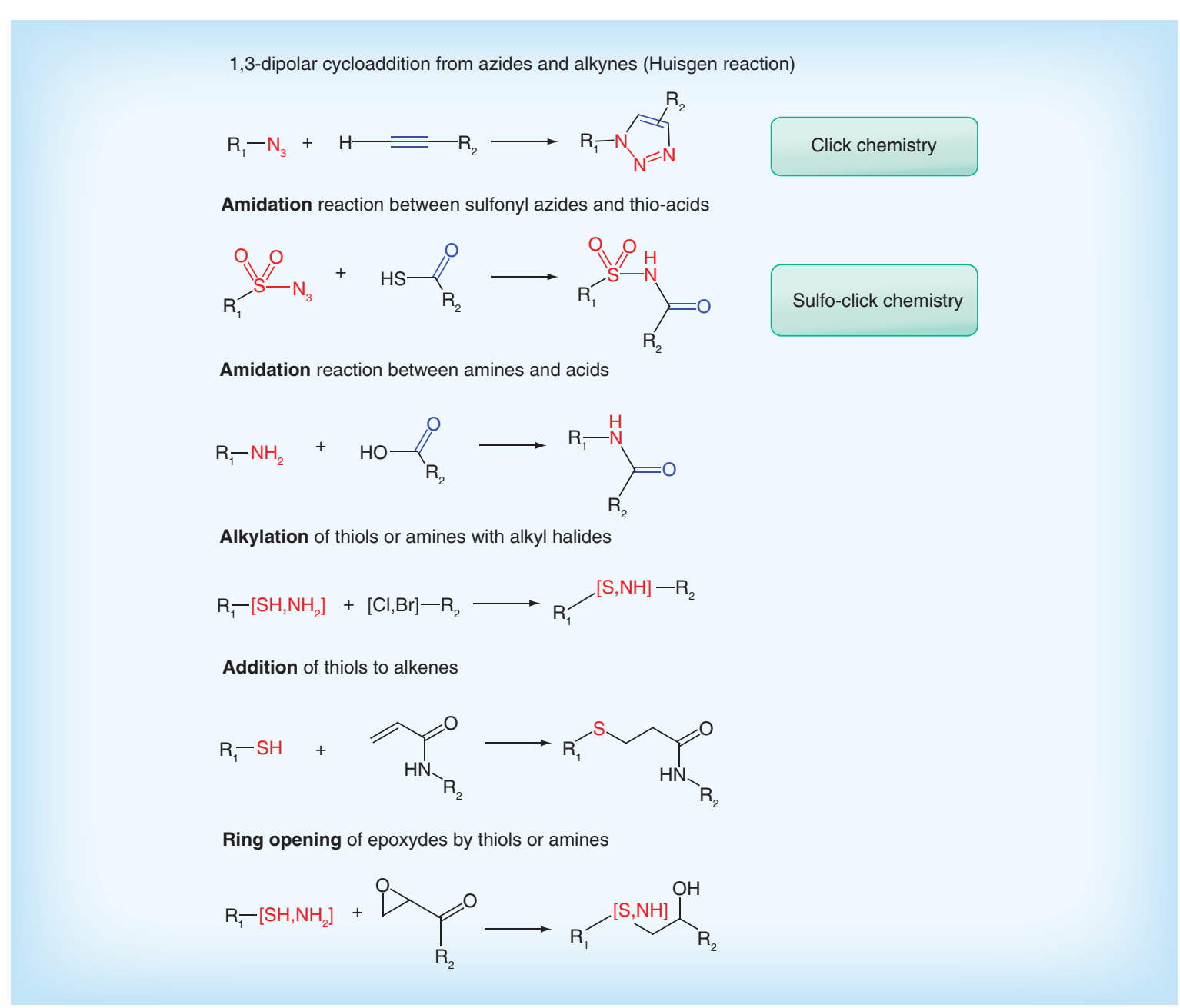

Figure 4. Chemical reactions amenable to kinetic target-guided synthesis reported in the literature. 
the KTGS [22]. Although acylation usually requires preactivated electrophilic carboxylic acid derivatives, Gelin et al. observed that amide formation between 5'-amino-5'-deoxyadenosine and carboxylic acid groups can be templated. The in situ amidation was also observed with citrate and a 5 '-amino$5^{\prime}$-deoxyadenosine bound into the active site without prior activation of the acidic moiety.

Alkylation of thiols by halides was also used as a KTGS reaction. Chase described the templated $\mathrm{C}-\mathrm{S}$ bond formation between bromoacetylcarnitine and coenzyme-A ( $\mathrm{CoA})$ by the enzyme carnitine acetyltransferase [52]. The formation of bound $S$-carboxymethyl-CoA carnitine ester leads to the formation of an inactive species that inhibits the enzyme. More recently, thiol alkylation using electrophilic alkyl chlorides and a mercaptoarylsulfonamide was used to find carbonic anhydrase inhibitors [38].

Thio-Michael addition between nucleophiles and electron-deficient acceptors has been used for both DCC [53] and KTGS [33]. Oueis et al. have used acrylamidecontaining huprine reagents and thiol-containing tetrahydroisoquinoline derivatives in a KTGS templated by $m \mathrm{AChE}$. As the high reactivity of thiols can lead to disulfide by-product, they took advantage of the esterase activity of AChE for a new 'two-step' KTGS: deacetylation of a thioacetate (deprotection 'in situ' of thiol precursors) followed by in situ thio-Michael addition [33].

Finally, the SN2 thiol opening of epoxide ring fills click-reaction criteria as it is stereospecific and quantitative. A KTGS templated by $14-3-3$ protein used this reaction. Epoxide-containing fusicoccin derivatives and thiol-containing pentapeptides (QSYDC) reacted in the presence of this protein to give ligands [27].

\section{Practical aspects of KTGS}

\section{Reagents \& protein concentrations}

Table 1 summarizes experimental conditions reported in the literature. Usually, KTGS takes place at $37^{\circ} \mathrm{C}$ for at least $24 \mathrm{~h}$. Some reported times are above 1 week. For the ligands to be detected, the concentration of reagents is usually high (from 3 to $600 \mu \mathrm{M}$ ). Also, the concentration of biological target needs to be near-stoichiometry (from 0.37 to $320 \mu \mathrm{M}$ ), thus impacting the size of the KTGS experiment due to the high requirements in protein amounts.

\section{Analytics}

Analysis of reaction mixtures is a critical step as expected conversions are low. Some efforts have been made to optimize the detection of ligands thanks to MS techniques. The first KTGS made use of MALDI-DIOS [5]. Subsequent use of LC-MS-SIM methods improved detection. More recently, the use of LC-HRMS-TOF [18] made possible to confirm the molecular formula of detected hits as well as diminishing the level of detection while still not being quantitative. Interestingly, Gelin et al. used x-ray cocrystallography to detect adducts [22] or bound reagents [54].

Historically, some authors claimed that there was a correlation between signal and affinity [14,30]. Recent papers show that ligands are indeed identified by KTGS, but their inhibitory potency (in the case of enzyme inhibitor) cannot be straightforwardly deduced from the KTGS experiment [18]. For example, hIDE templates the synthesis of both syn- and anti-triazoles ligands with the same LC-MS signal but 1,4-triazoles are the most potent as inhibitors (as demonstrated by ${ }^{19} \mathrm{~F}$ NMR afterward)

In any case, it is likely that improvements of both sensitivity and selectivity of analytical techniques will continue to lower the amount of falsely negative results.

\section{Biological controls}

An important point in KTGS is the use of controls (Table 1). To assess the specificity of the templated reaction, KTGS without the target is usually performed as negative control (buffer). Some publications report the use of bovine serum albumin, though this protein is not the best control as it can template itself reactions [55].

Recently, specific protein mutants have been developed as controls. Bcl-XL ${ }^{(\mathrm{F} 131 \mathrm{~A}, \mathrm{D} 133 \mathrm{~A})}$ and $\mathrm{Bcl}-\mathrm{XL}^{(\mathrm{R} 139 \mathrm{~A})}$ mutants with a lower affinity for inhibitor ABT-737 were used as controls of KTGS Bcl-XL-templated reactions to find new inhibitors binding at the ABT737 site [25]. As well, mutation H223E in $L m$ NADK was used as a negative control of the in situ generation of a ligand [22]. Orthologous protein from a different species has also been used as positive control. ACh-binding protein from Aplysia californica is less efficient to promote the formation of a ligand than its Lymnaea stagnalis counterpart. One of the hypotheses is that these two homologous proteins differ at an amino acid of the complementary subunit (Tyr 55 for A. californica and $\operatorname{Trp} 53$ for L. stagnalis). $\operatorname{Trp} 53$ in $L$. stagnalis better stabilizes the cation $-\pi$ interactions between the aromatic ring and the quaternary amines of ACh mimics than Tyr55 in A. californica. LsAChBP (Y55W) was produced and was shown to restore partially the templating capabilities of AChBP from A. californica [14]. In the same line, a recent example by Abell et al. reports the design of a mutant of $S$. aureus biotin protein ligase $\left(S a \mathrm{BPL}_{\mathrm{R} 122 \mathrm{G}}\right)$ to enhance the turnover rate for the KTGS reaction to improve efficiency and sensitivity. Indeed, the Arg122 was shown to be implicated in the stabilization of the 


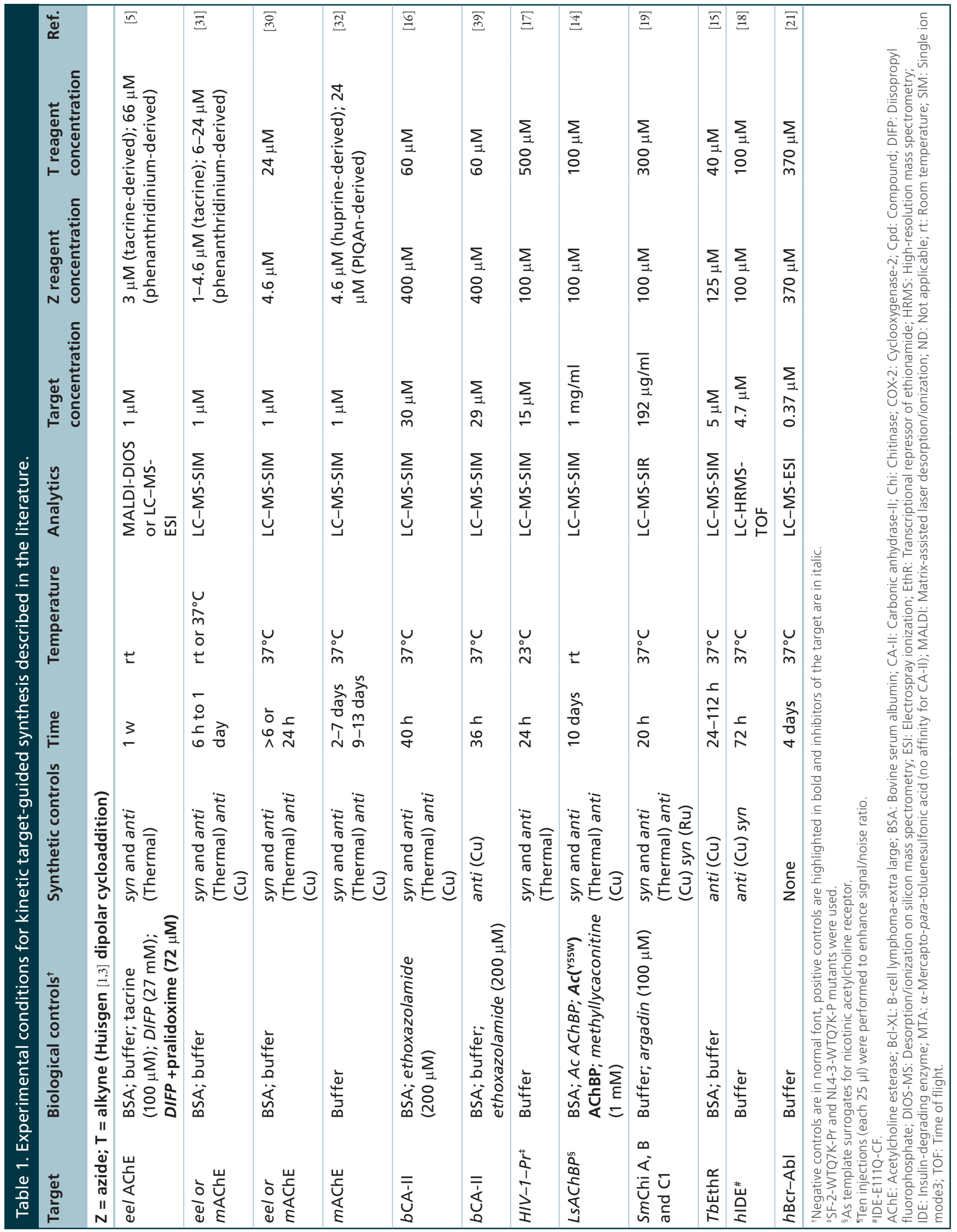




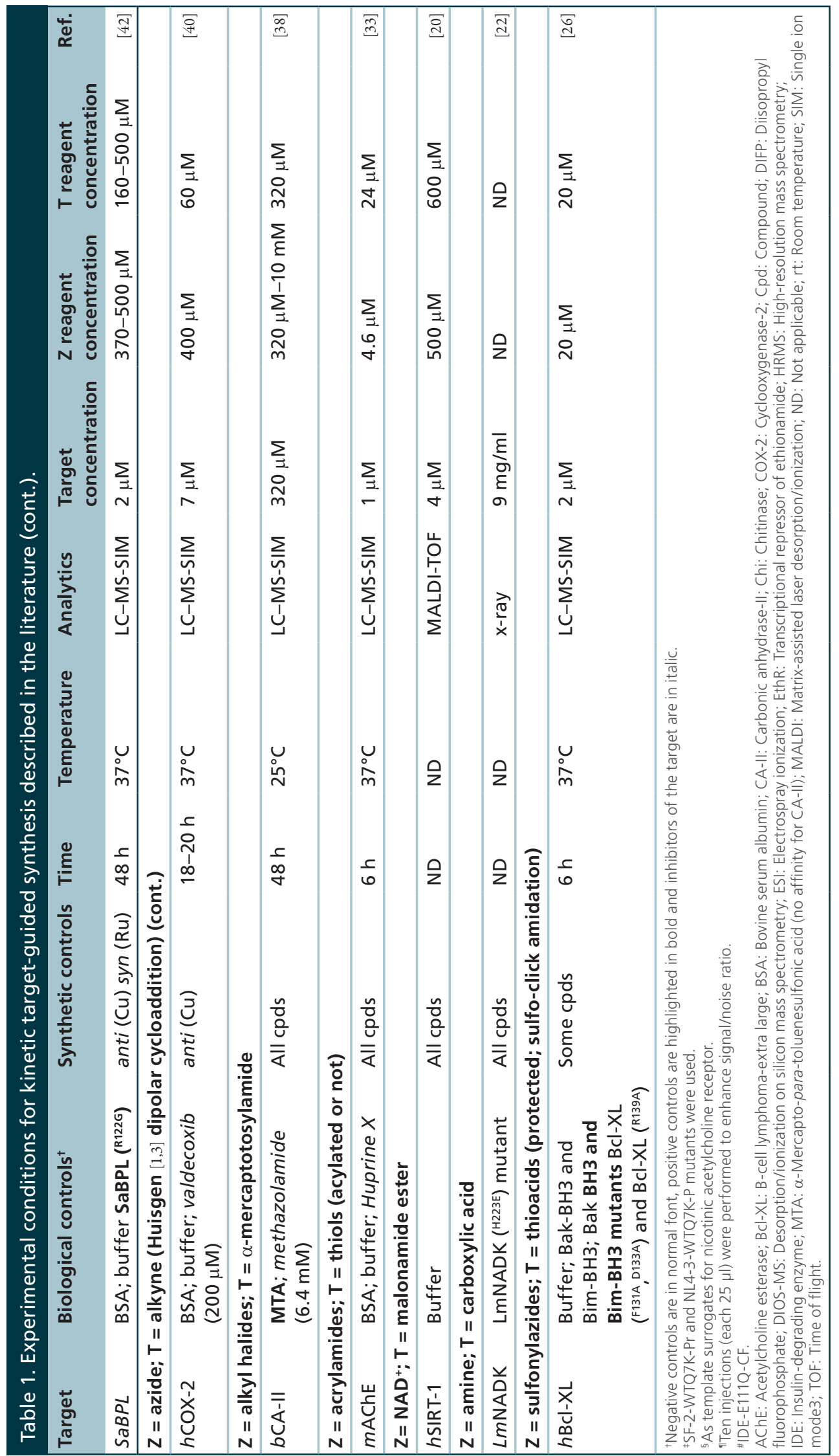


closed conformation of this enzyme, thus preventing a rapid dissociation of the ligand from the binding site and a good turnover of the enzyme. The mutation of this amino acid does not affect the KTGS and the binding site of the ligands but enhances the turnover [42].

Another strategy consists in using known ligands to compete with the templated compounds. For enzymes, reference inhibitors used are: diisopropylfluorophosphates (DIFP) - a general serine esterase covalent inhibitor or huprine-X for AchE [5], ethoxazolamide for CA-II [16], valdecoxib for COX-2 and argadin for chitinase [19]. Interestingly, the use of pralidoxime - a detoxification agent of organophosphates - can be used to restore DIFP-inactivated AChE KTGS [5]. In these experiments, a structurally related, nonbinding analog of the inhibitor (such as $\alpha$-mercapto- $p$-toluenesulfonic acid for benzenesulfonamides inhibitors of CA-II) can be used to show that the inhibition of KTGS by the genuine inhibitor is pocket-specific.

For other targets like protein-protein interactions, the native partner of the protein used for KTGS can be used as a control. For example, $\mathrm{Bak}_{-} \mathrm{BH}_{3}$ and $\mathrm{Bim}-\mathrm{BH}_{3}$ peptides were used as negative controls for the KTGS of ligands by Bcl-XL [26]. On the contrary, mutated Bak- $\mathrm{BH}_{3}$ and $\mathrm{Bim}-\mathrm{BH}_{3}$ peptides that have a lower affinity for $\mathrm{Bcl}-\mathrm{XL}$ can be used as positive controls [26].

Negative and positive controls for KTGS can be obtained by using either mutant proteins or small compounds (inhibitors, for example). The former requires however the knowledge of the protein $3 \mathrm{D}$ structure and a protein production capability. The latter requires the knowledge of structure-activity relationships.

\section{Synthetic controls}

To be sure that the peak in LC-MS corresponds to the clicked compound, synthetic controls were used to support the KTGS proof-of-concepts, though highresolution MS-TOF provides good hypotheses on the molecular formula of the observed compound [18].

Many published KTGS provided most or all synthetic controls (Table 1). Also, in in situ click chemistry, where anti-(1,4) and syn-(1,5)triazoles can both result from the reaction of azides and alkynes, synthetic controls were used to assess the regioselectivity of the templated reaction. When possible mixture of syn-and anti-controls are produced via the thermal reaction [5]. Attribution of both peaks to a specific isomer can be made by synthetizing explicitly the anti-regioisomer via a Cu-catalyzed reaction [56,57] while the syn-isomer can be obtained via Ru-catalyzed reaction $[42,58,59]$. Nevertheless, these reactions are not applicable to all starting materials and other retrosynthetic pathways to access syn-controls may be needed [18].

Finally, the synthesis of mixtures of synthetic controls is of particular interest in the case of multicomponent KTGS (see below) [15,18].

\section{Implementation of KTGS experiment: size \& format}

Three different formats are reported (Figure 5 \& Table 2). About half of the examples consist in binary mixtures of $\mathrm{Z}$ and $\mathrm{T}$ reagents that are presented to the target in the same vessel. One combination is thus evaluated at a time (Figure 5A). Though this allows direct attribution of hit structures, it is rather time and resource consuming as large quantities of protein target and many LC-MS analyses are required.

To make the process more efficient, a multicomponent setup can be used (Figure 5B) where at least one partner (e.g., T reagents) is presented to the target as a mixture of competing reagents rather than a single entity. This reduces both the amount of enzyme needed and the duration of LC-MS screening. Interestingly, analytical controls can be done in clusters too. This strategy has been so far applied only to AChE [30], CA [16], HIV protease [17], AChBP [14] and EThR [15]. The largest size for clusters of reagents is 10. One of the caveats for that format is that reagents are in competition with each other and though it may form ligands, false positives may occur. Also, selectivity and sensitivity of the LC-MS/SIM technics are critical, and the use of isobaric reagents in the same mixture should be avoided.

A recently introduced variant of the multicomponent setup involves orthogonal partitioning (Figure 5C) [18]. In example published, alkynes were sorted in two orthogonal partitions (A and B), which were used in two parallel TGS experiments. A distinctive feature of orthogonal partitioning is that a given cluster from partition A has only one alkyne in common with any cluster of partition B, in strict analogy with orthogonal combinatorial libraries. Initially, these libraries were developed for structural elucidation of active compounds within combinatorial mixtures [60]. This variant gives better chance of any alkyne to find its way to the binding site within a mixture, since it is presented to the target in two different competing environments, maximizing the chance of templated reaction and detection of binding triazoles among all possible combinations, thus reducing false negatives while retaining the economy of multicomponent format.

A critical component of the orthogonal multicomponent setup is the design of reagent clusters. Deprez et al. 
(A) Binary (single component)

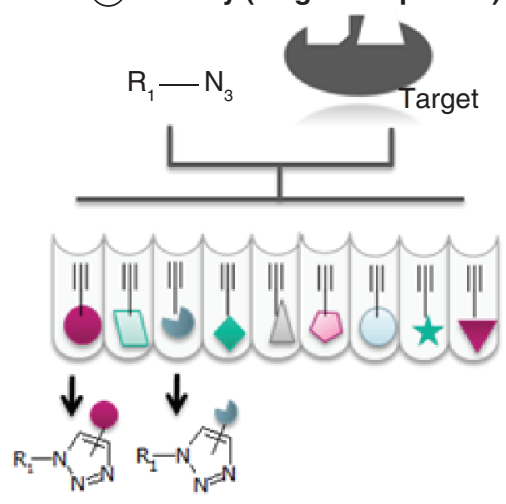

(B) Multicomponent

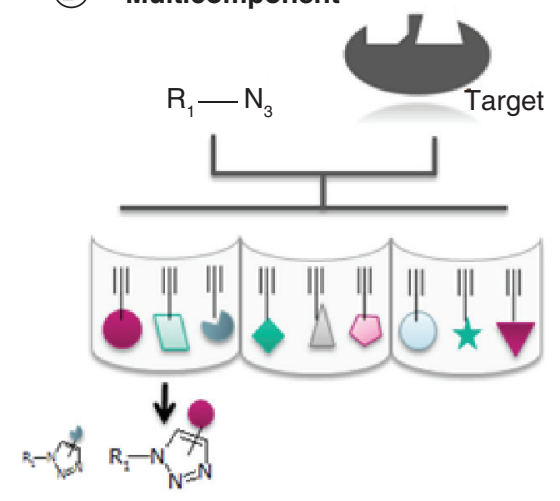

(C) Orthogonal multicomponent
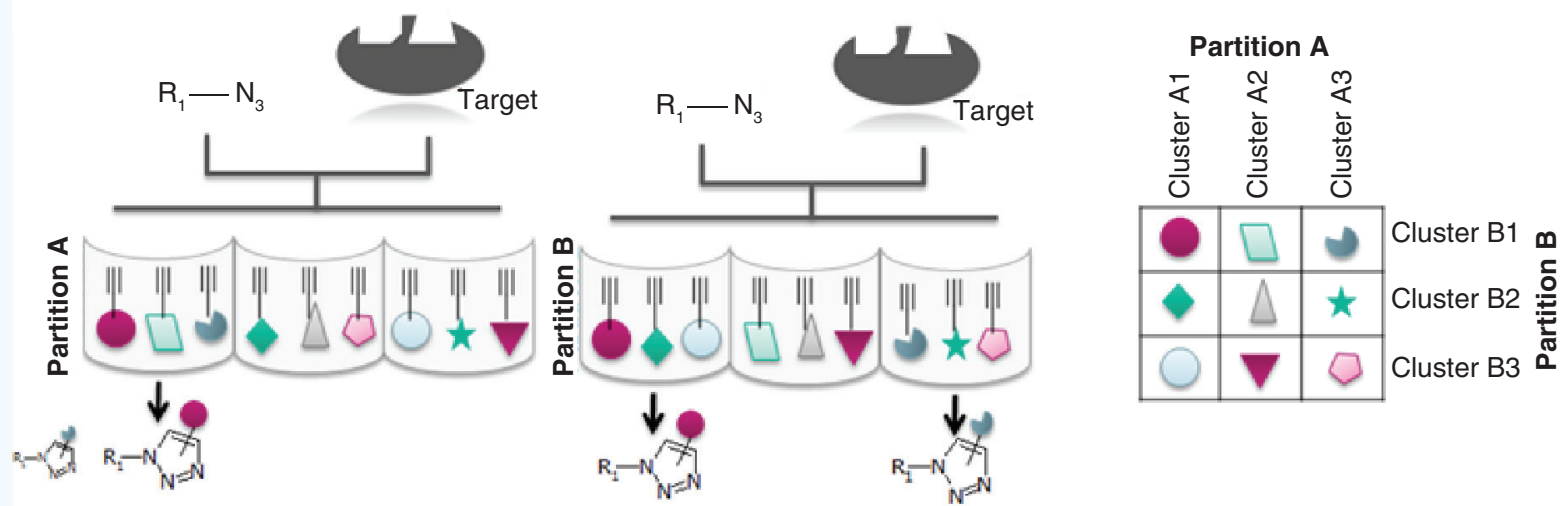

Figure 5. Kinetic target-guided synthesis strategies. In situ click chemistry is used as illustration. Z = azides and T = alkynes.

(A) Single binary experiment: each alkyne and azide react in binary mixture in the presence of the target. Two hits are detected.

(B) Multicomponent experiment: azides are presented as a single component while alkynes are presented to the target as mixtures (three clusters of three alkynes each). Due to competition between alkynes, only one triazole is detected (magenta sphere).

(C) Orthogonal multicomponent experiments: two orthogonal partitions A and B of alkynes are designed in such a way that competitors of a given alkyne are different when present in clusters from different partitions. Such orthogonal partition varies the diversity of environment and allows both hits to be detected (magenta sphere and gray sector). It decreases the number of false negatives while reducing the number of kinetic target-guided synthesis (KTGS) experiments. In the case of KTGS of (1*9) size, single KTGS proceeds in nine experiments (A), multicomponent KTGS with three clusters proceeds in three experiments (B) and orthogonal multicomponent KTGS with three clusters proceeds in $2 * 3$ experiments (C).

used two different partitions where alkynes are sorter in function of either the type of backbone (partition A) or the type of substituents (partition B) while making sure that no isobaric compounds were present in the same cluster [18].

In order to be advantageous in reducing the number of KTGS reactions, this strategy should fulfill the following criteria: (Number of reagents/Number of clusters in partition) $>2$. This thus applies to sets of reagents of at least nine, partitioned into three clusters. The more the sets of reagents are populated, the more advantageous is this strategy. For example for hIDE, using one azide and 90 different alkynes, 19 KTGS experiments were performed (instead of 90, if all binary syntheses were attempted).

More particularly, multicomponent formats using large sets of diverse alkynes have been used for AChE 46 compounds, EthR 120 compounds, Chitinase 71 compounds and IDE 360 compounds (Table 2). The set of azides is usually smaller due mainly to synthetic reasons. It is also most of the time attached to the warhead, so the diversity is not required at this part. 

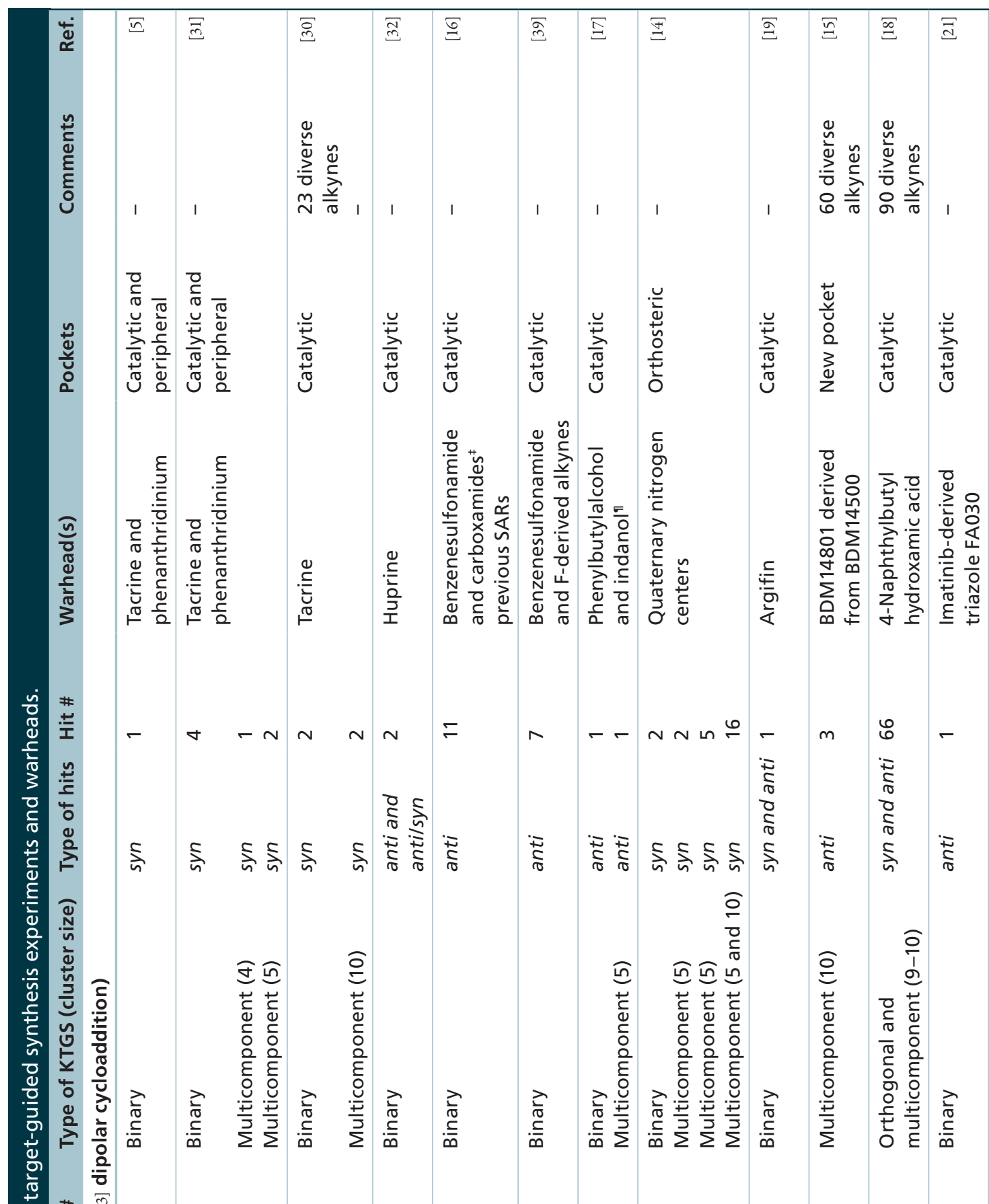

虫

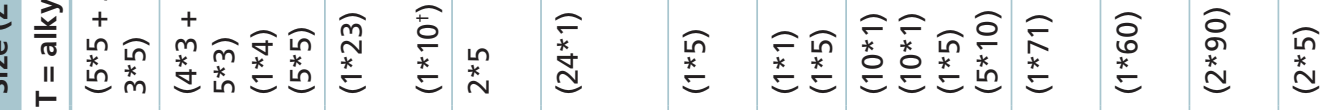




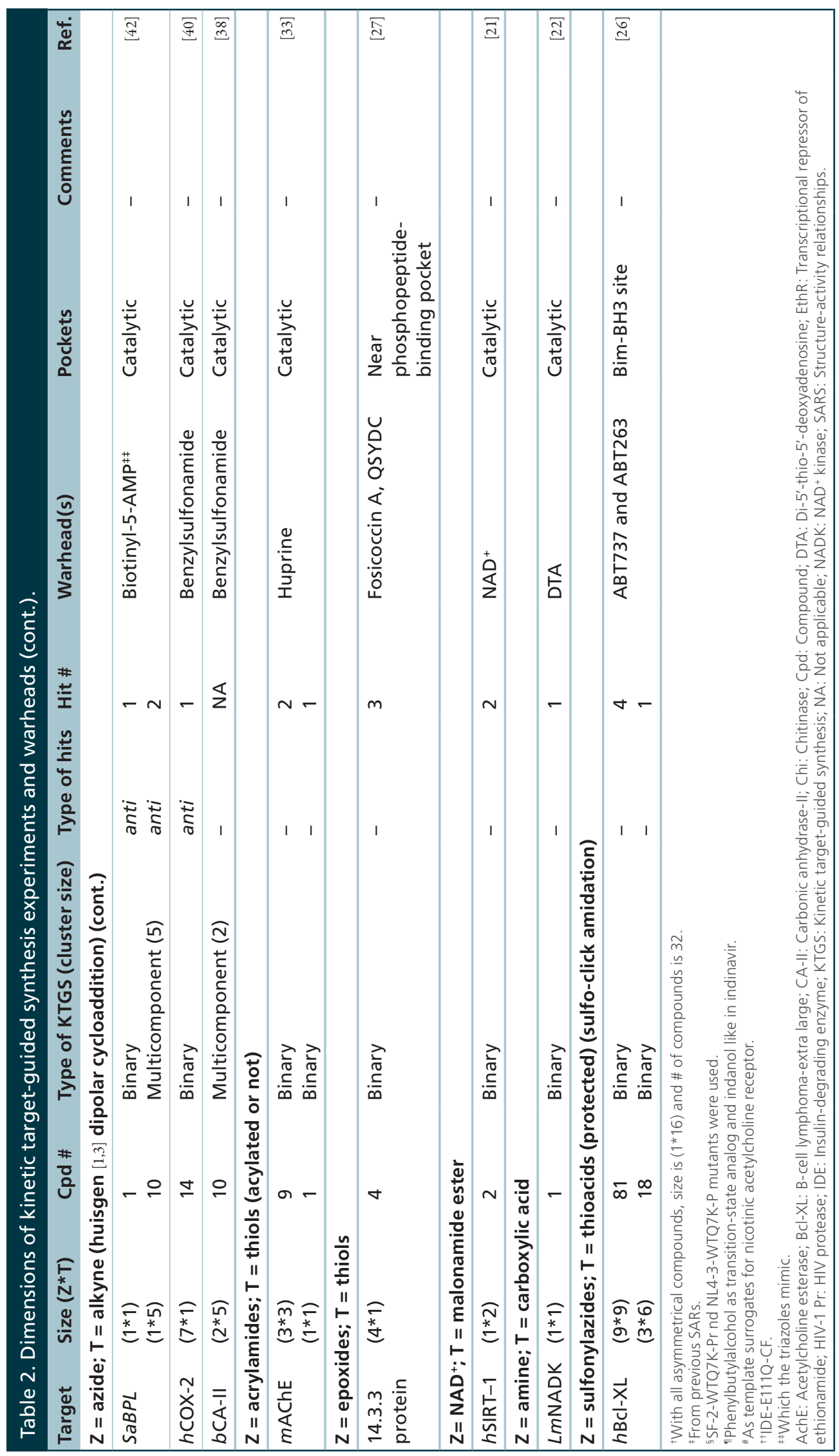


The Hit rate is quite variable since many experiments were done as proof of concepts. Interestingly, for IDE, the hit-rate was quite high and gave trustful preliminary SARs.

\section{Chemical design in KTGS}

\section{Reagents: are they fragments or not?}

Fragments usually display high solubility, a molecular weight $<300 \mathrm{~g} / \mathrm{mol}$; a number of hydrogen bond donors and acceptors $<3$ and a number of rotatable bonds $<3$. $x$-ray and NMR are used to identify binding modes of fragments for follow-up optimization by fragmentlinking or fragment-growing. Over the recent years, the advantage of screening fragments has been proven. Reasons for success are: first, that LE is higher for fragment and thus, adding hydrophobic groups during hit-to-lead does not add MW penalty; second, that fragments are small, thus they fit a large variety of binding pockets [61].

Interestingly, in KTGS, reagents do not need to be fragments. They are usually much bigger molecules with MW above 300, as in several cases, they bear two functionalities: a warhead to bind to a specific pocket of the protein, and a reactive function to accomplish the coupling to the second reagent. As a result also, they usually display more than three hydrogen-bond donors or acceptors.

Fragments usually display high LE but low affinity [61]. The first disclosed KTGS made use of potent
(10-100 nM) reagents containing a tacrine moiety, critical for interaction with $\mathrm{AChE}$. These reagents gave triazoles in the femtomolar range [5]. For chitinase, azide warheads, derived from argifin, displayed $\mathrm{IC}_{50} \mathrm{~s}$ of 0.045 and $0.58 \mu \mathrm{M}$ [19]. More recently, many examples report lower affinities for the warheads, while giving successful results in KTGS. Another example of AChEtemplated reaction used micromolar range phenyltetrahydroisoquinoline [31]. For insulin-degrading enzyme, azide precursors displayed an $\mathrm{IC}_{50}$ of $200 \mu \mathrm{M}$ for the target [18]. For the Bcl-XL-templated reactions, the thio-acids or sulfonyl azides did not show any inhibition up to $100 \mu \mathrm{M}$ concentrations [26]. In conclusion, KTGS reagents are of varied size and starting affinity. In many reported cases, one of the reagents was specifically designed to interact with the target, and therefore, did not match the fragment definition.

\section{Templated compounds: are they the best inhibitors?}

Several publications mention a possible correlation between the amount of a product formed by KTGS and its inhibitory potency [6,14]. It seems to be the case for the reactions templated by AChE or AChBP where the reagents used displayed a very high affinity themselves for the target. These observations should be balanced by a reminder that most detection techniques used in KTGS are not quantitative. Moreover, they lead to the mislead-

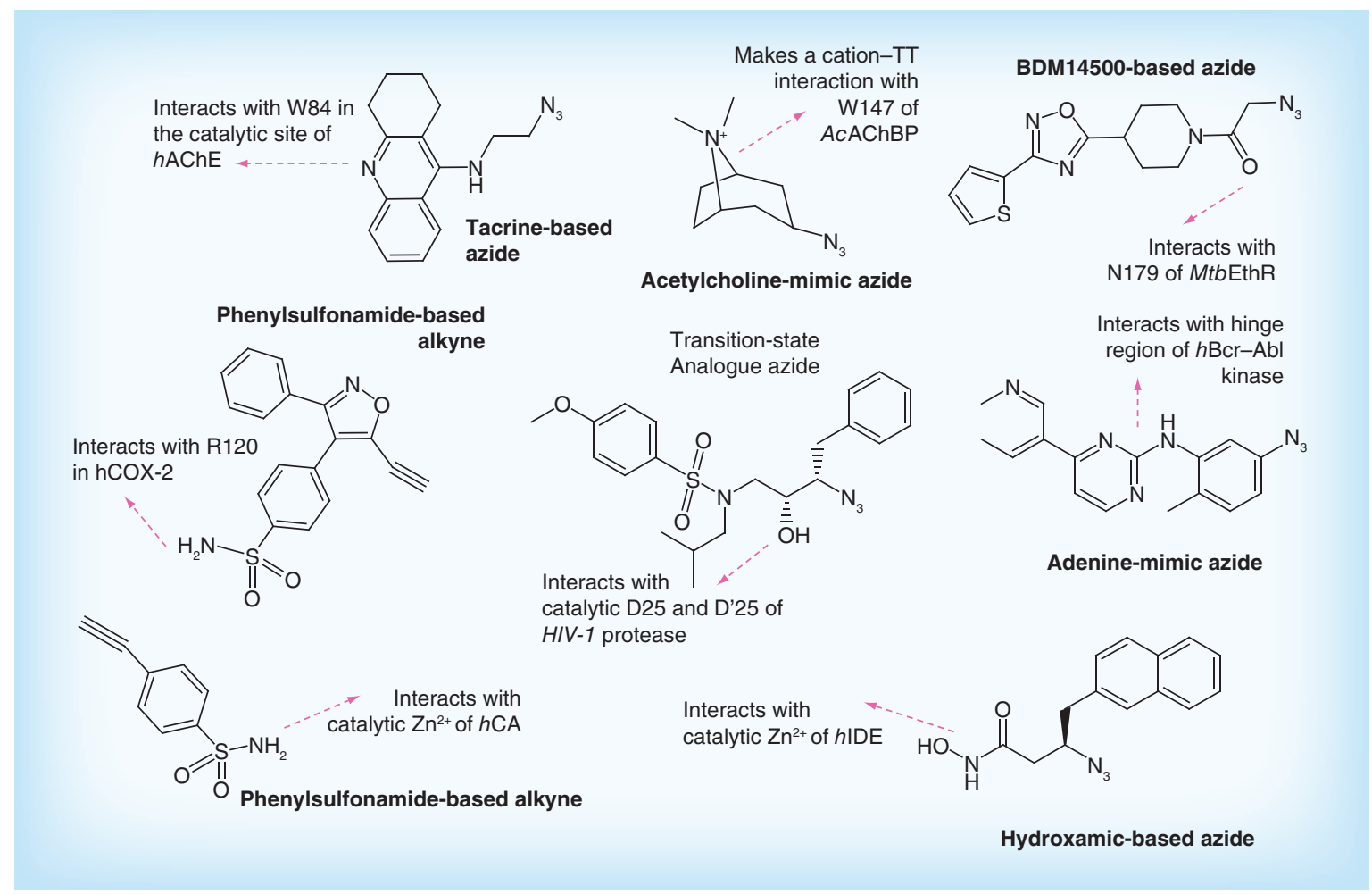

Figure 6. Warheads examples. 
ing concept that KTGS selects the best inhibitors while it selects only the best ligands with improved affinity. For instance, IDE has catalyzed the formation of both 1,4and 1,5-triazoles ligands but 1,4-triazoles are very good inhibitors while 1,5-triazoles are not [18]. One should also keep in mind that KTGS is a kinetically driven selection process. Therefore, unless the reaction products are structurally and 'pharmacophorically' very similar to the transition state bound to the protein, it is not guaranteed that the selected product will be the most potent.

\section{How to select a warhead?}

In KTGS, one of the reagents can be considered as a warhead (Table 2 \& Figure 6) to direct the templated ligands within the target. To design such warheads, one needs to use previously known structure-activity relationships. For enzymes, the warhead usually targets the catalytic site.

For example, the warhead used for HIV protease is directly inspired from indinavir, a marketed drug that mimics the transition state of the enzyme [17]. Sets of azide- and alkyne-reagents based on the known sitespecific inhibitors tacrine (that binds the active site) and phenylphenanthridinium (that binds the peripheral site) were developed for the KTGS by AChE to identify 'divalent' inhibitors of this enzyme $[5,32]$. For the Bcl-XL/BIM protein-protein interaction, the warhead was derived from a known reference inhibitor (ABT737). While many examples derive from small molecules, some derive from larger natural compounds like argifin [19], fusicoccin or peptides [27]. In the case of AChBP, trimethylammonium-containing reagents mimicked ACh and maintained a crucial cation- $\pi$ interaction with a tryptophan.

Along with information about the binding pockets, some publications report the use of cofactor as an initial idea to design a warhead. An example is the use of CoA-derived reagents for the carnitine acetyltransferase-templated KTGS [62]. Another example is the use of $\mathrm{NAD}^{+}$as inspiration for sirtruin-templated KTGS [20].

It should be noted that the actual binding of the templated ligand may eventually be different from the initial warhead idea and the known structure-activity relationships. For example, the $\mathrm{x}$-ray diffraction of BDM44768 bound to hIDE revealed that its naphthyl group does not interact in a way consistent with previous SAR [18].

\section{What about linkers between warheads \\ $\&$ reactive functions?}

KTGS proceeds by reacting reagents bearing each a reactive function and for at least one reagent, a warhead. Interestingly, several examples mention the need of optimizing reagents by adding linkers of various lengths between the reactive function and the warhead to allow some flexibility for the KTGS to occur. In AChE-templated reactions, where two different binding sites are targeted (catalytic and peripheral), authors have varied the chain lengths of their huprine-derived acrylamides and a length of three templated the reaction while lengths of two and four did not [33]. Also, a length of three methylene moieties between fusicoccininspired warhead and epoxide function was found to be critical to template the ligand formation by $14-3-3 \zeta$ protein $(+199 \%[27])$.

\section{Templated ligand/protein complex: binding \& conformations \\ Exploring conformations}

It is noteworthy that one of the advantages of the KTGS over classical-led discovery techniques, including fragment-based drug discovery, is its ability not to hinder protein flexibility and, on the contrary, to allow the identification of unknown or less abundant conformations.

Fokin $e t$ al. mentioned that the reagents may even drive a preferred conformation rather than bind to a preexisting conformation of the unbound protein [14].

Also, it was demonstrated for AChE that the synpreference for the triazole is due to the change of conformation of $\operatorname{Trp} 286$. This amino acid then is involved in a $\pi$ interaction with the phenanthridinium of the inhibitor, located at the peripheral anionic site of AChE. Trp286 points also to Leu289 and the solvent [63]. This new conformation impacts the cell-adhesion properties within synapses of AChE.

For MTbEthR, Willand et al. have unveiled a new 'open-gate' conformation that impacts the transcriptional repressor capabilities of the protein. Interestingly, the final triazole cocrystallization with MTbEthR revealed the unexpected flip of Phe114 and Phe184 that extend the hydrophobic channel within the protein, whereas the azide precursor does not [15].

BDM44768 inhibitor of hIDE interacts with the catalytic site formed by both IDE-N and IDE-C thus keeping the enzyme in a closed, inhibited conformation. The hydroxamate chelates the $\mathrm{Zn}^{2+}$ in the $\mathrm{N}$-terminal domain and the para-fluorophenyl group stacks with Phe820 of the C-terminal domain [18].

Finally, KTGS allows the exploration of interactions and intersubunit binding sites of an oligomeric protein as was shown, respectively, by Manetsch and Fokin [26,14]. This illustrates how protein interfaces can also be explored via this strategy.

\section{Triazoles as pharmacophores}

Triazoles participate in binding of the ligands and are not just 'linkers'. Triazoles produced are capable of 
(A)

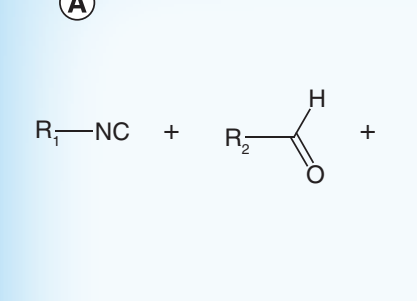<smiles>N=C(N)c1ccc(N)cc1</smiles>
Thrombin-CLEC
Tris-Buffer ( $\mathrm{pH} 8.3)$
Water/iPrOH (2/3), 1 week

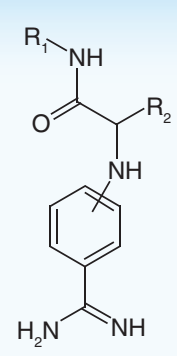

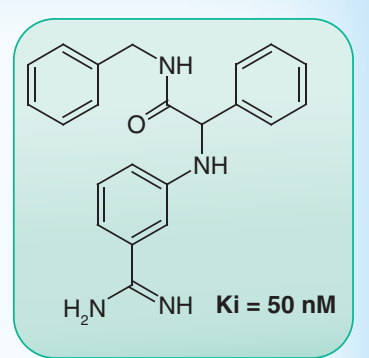

(B)

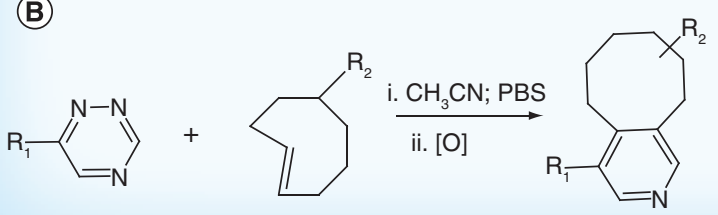

Figure 7. Other reactions for kinetic target-guided synthesis. (A) The $3 C$-Ugi reaction has already been used to find thrombin inhibitors via kinetic target-guided synthesis and (B) bioorthogonal 1,2,4-triazines, electrondeficient dienes, could be tested as reagents for kinetic target-guided synthesis, in inverse-electron-demand Diels-Alder reactions.

establishing a wide range of interactions, such as dipolar interactions, hydrogen bonding, $\pi$-stacking interactions and cation $-\pi$ interactions. Also, it has been proposed as an amide bond surrogate, for example, in an analog of imatinib $[64,65]$. In the inhibitor of BPL, the triazole interacts via several key interactions to stabilize the enzyme [66]. The N2 and N3 are implicated in hydrogen bonds with Arg125 (side chain) and Arg122 (backbone) while the C5 interacts via its hydrogen with Asp180. Also a T stacking is observed with the aromatic amino acid Trp-127. In AChBP, the triazole was used as a surrogate of the ester HBA part of ACh. It binds via a water molecule Ser203 on the protein [14]. In the MTbEthR inhibitor BDM14801, the triazole ring interacts via van der Waals forces with Phe110 and Trp145 in the channel of the protein [15] and binds to a pocket that did not exist in the complex between the warhead alone and the protein, showing that KTGS can explore the conformational space of the protein.

In hIDE inhibitor BDM44768 [18], the triazole makes a beneficial interaction with the positively charged guanidinium of $\operatorname{Arg} 824$ at IDE-C. This is consistent with the high affinity of triazoles for cations [67]. As a consequence, the isosteric replacement of the 1,2,3-triazole by a 1,2,4-oxadiazole led to less potent inhibitors. Indeed, the value and direction of the dipole moment are quite different for the two heterocycles (3.55 Debye for triazoles; 1.2 Debye for oxadiazoles) [68].

\section{Future perspective}

KTGS has been applied to several drug discovery relevant proteins and proved to be a genuine hit-finding technique. Already two publications disclose the use of the identified templated ligands as tools for in vivo studies $[18,39]$. To warrant a wider application of KTGS in drug discovery and chemical biology, several aspects of this chemistry deserve further investigation.

\section{Find other KTGS amenable reactions}

Several click reactions have been disclosed and used in cells for protein labeling, for example [8]. As they are catalyst-free, these reactions are good candidates for KTGS and could serve to identify protein ligands.

Also strategies aiming at optimizing the selectivity of the KTGS to avoid undesirable side reactions will add valuable tools for medicinal chemists. Manetsch and colleagues have already disclosed the use of protected thiols that are deprotected using biocompatible media to allow subsequent sulfo-click reaction [69]. Sabot $e t a l$. have ingeniously used the esterase activity of AChE to release the reactive function of their KTGS precursors [33].

So far also, KTGS uses only two sets of reagents. An interesting approach would be to use several sets of reagents by developing the use of multicomponent reagents. For example, Weber et al. have used the multicomponent reaction to identify nanomolar inhibitors of thrombin (Figure 7A) [70]. This templated reaction is a mix between DCC (as the first step involves the reversible imine formation between the amine and an aldehyde) and KTGS as the final rearrangement is irreversible. Other reactions that work in water could be used also [71].

New reagents could be also explored. Recently, Kember $e t$ al. disclosed the use of triazines as bioorthogonal 
reagents with improved properties in comparison with tetrazines (Figure 7B) [72].

\section{Target other protein of pharmaceutical interest} It makes no doubt that some classes of enzymes (such as hydrolases) and soluble receptors make suitable protein partners for KTGS. However, several classes of protein targets of high interest to the drug discovery community still need further work to be considered as valuable targets of KTGS. In the coming years, a confirmation of the usefulness of KTGS for the discovery of inhibitors of protein-protein interactions or kinases by new examples is necessary.

A yet untouched class of important drug targets is membrane-bound receptors, such as G-protein-coupled receptors (GPCRs). The main technical challenge with integral proteins would be the use of membranes, probably overexpressing cell lines and the development of the suitable biological controls (like cells transfected with a mock protein). Known pharmacophores for GPCRs could then be used to design warheads.
Many articles report the structure-activity relationships for triazole-derived bioactive compounds that were discovered by 'classical' click chemistry [73]. This information could help the design of KTGS to allow the identification of new compounds of interest acting on different proteins.

\section{Playing with natural compounds \& peptides}

Templating a coupling reaction on natural compounds could be of great interest. Some already published examples are adenosine-derived [22] or biotin-derived ligands [42]. Peptides could also be explored. In situ click chemistry was elegantly associated with the synthetic epitope-targeting technique by Heath et al. to identify peptidic ligands specific for a distinct region of proteins (Figure 8). In this approach, an azide- or alkyne-containing epitope is chemically synthesized and used as both a catalyst and a reactant for the in situ click chemistry reaction. This peptide is screened against a one bead, one compound (OBOC) library [74], containing peptides bearing the comple-
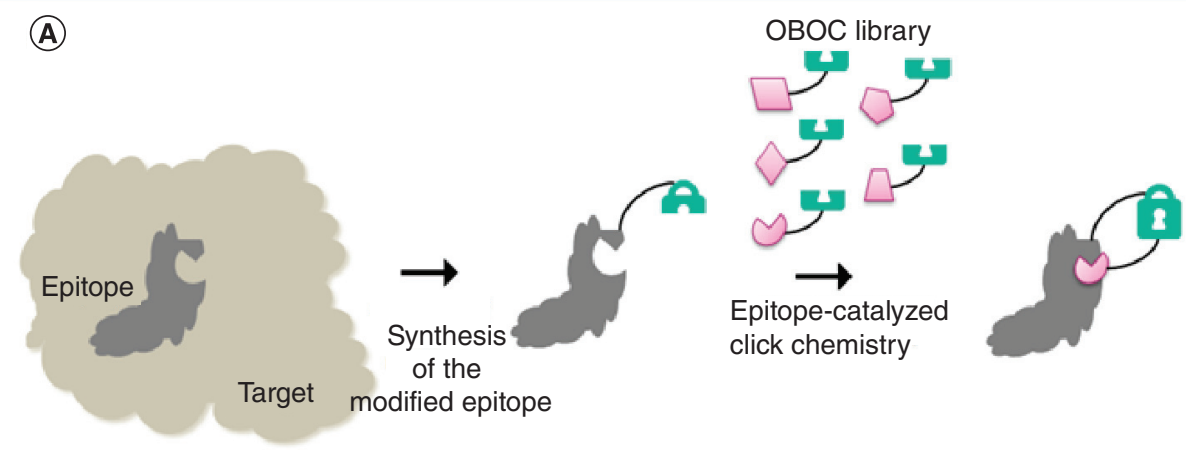

(B)
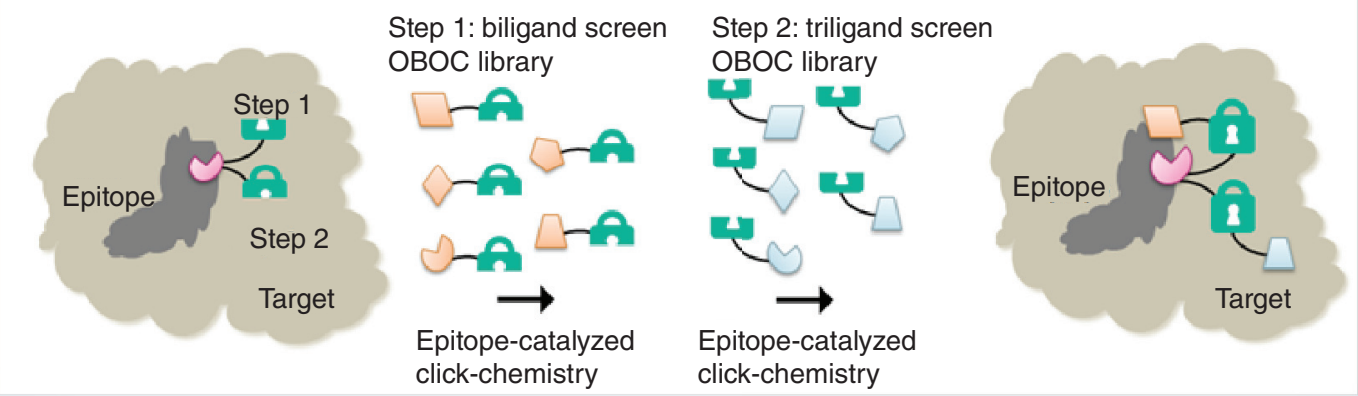

Figure 8. In situ click chemistry associated with epitope targeting strategy. (A) A region of the domain of interest is chemically synthesized as a modified epitope bearing a clickable moiety (e.g., alkyne or azide). An OBOC library bearing the complementary moiety for the click reaction is screened against the epitope to discover a binder in close proximity to the domain of interest (e.g., active site or site of mutation). (B) The discovered binder is used as an anchor and a clickable moiety is appended for the first in situ click chemistry screen to afford a biligand. A clickable moiety is then introduced into the biligand for the second in situ click chemistry screen to identify a triligand.

OBOC: One bead, one compound. 
mentary moiety to complete the click reaction to identify the peptide binder. Then, using the full protein as the target, the discovered peptide binder could be then used as an anchor ligand to identify extended, more potent, ligands (Figure 8). This strategy was successfully implemented with two Akt kinase isoforms.
For Akt2, the most potent triligand displayed a 20 $\mathrm{nM}$ affinity [75], while for Akt1 a $\mathrm{K}_{\mathrm{D}}$ of $54 \mathrm{nM}$ with a 10:1 selectivity for the E17K PH domain of Atk1 compared with the wild type was obtained [76]. Thanks to this ligand, Heath et al. could attach a dye to the protein target that is useful for imaging and to syn-

\section{Executive summary}

\section{Background}

- Target-guided syntheses include in situ dynamic combinatorial chemistry and kinetic target-guided synthesis (KTGS).

- KTGS uses irreversible reactions templated by the protein.

- The in situ click chemistry is the most widely used KTGS strategy.

Biological applications of KTGS

- Many biological applications have been described so far.

- Therapeutic areas range from infectious diseases, CNS, cancer or metabolic diseases.

- Biological targets are soluble and compass many enzymes (of all classes), receptors, ligand-gated channels and protein-protein interactions.

- Enzymes used in KTGS are mainly proteases and other hydrolases including esterases.

- A total of almost 30 KTGS have been disclosed so far.

- An inhibitor of CA-II has been used in vivo PET imaging.

- The first catalytic-site inhibitor of insulin-degrading enzyme (IDE) to be used in vivo for both pharmacokinetic and pharmacodynamics studies has been discovered by KTGS.

Click synthetic strategies

- A limited number of click reactions have been used so far for KTGS.

- These reactions should be biocompatible.

- Most examples make use of the Husigen dipolar cycloaddition.

- Some other examples use thiol-based reagents.

Practical aspects of KTGS

- Key aspects of KTGS include the concentration of reagents and protein, which are usually high and the time of reaction that can vary from a few hours to weeks.

- Detection of templated ligand is done by LC-MS techniques.

- A wide variety of biological controls have been disclosed from buffer, small molecule positive or negative controls and specific protein mutants.

- The largest KTGS performed allowed the potential synthesis of 360 ligands.

- Three different formats of KTGS exist with complementary advantages/drawbacks: binary, multicomponent and orthogonal multicomponent.

Chemical design aspects of KTGS

- Reagents used in KTGS do not fulfill the fragment criteria.

- KTGS does not require prior knowledge of the protein structure (x-ray or nuclear magnetic resonance) in strict opposition with fragment-based drug discovery.

- KTGS identified ligands but no information on the activity can be directly deduced.

- Warhead design needs prior structure-activity information.

- A variety of linkers should be investigated in sets of reagents as flexibility of reagents binding is important.

Templated ligand/protein complex: binding \& conformations

- In opposition to fragment-based drug discovery that uses fixed protein structures, KTGS can allow to explore protein conformations that are unexpected and not captured by x-ray diffraction.

- In the case of in situ click chemistry, many examples mention that the triazole ring is not just essential for bridging reagents but is a key element of pharmacophore implicated in various types of interactions.

Future perspective

- Future efforts should be made on expanding the pool of KTGS amenable reactions including the appealing multicomponent reactions.

- Other druggable proteins like membrane-bound proteins should be explored.

- KTGS could be used to identified peptides and pseudopeptides.

- A better balance between protein amounts and mass detection should be found.

- A cellular version of KTGS could be developed.

- Open-source collection of azides and alkynes could help expanding the scope of KTGS. 
thesize by in situ click iteration a triligand that blocks the interaction of the PH domain of Akt1 with PIP3.

In the context of the search of nontraditional drugging approaches, this technique was also successfully performed on Botulinum neurotoxin serotype A (BoNT/A), the most lethal known toxin. [77] The direct inhibition of its active site is prevented because of its occlusion by a belt subdomain. To circumvent this, an epitope-targeting strategy combined with in situ click chemistry was used to find a macrocycle that binds to an active site-adjacent epitope of BoNT/A. Then, a second in situ click chemistry assay was performed in the full-length BoNT/A to bind the first macrocycle to another competitive inhibitor discovered previously by rational design affording a divalent inhibitor. This inhibitor acts as a Trojan horse as it is carried into the neuronal cells by the holotoxin. Then, the active site is exposed inside the cytosol and the substrate-mimicking inhibitor moiety can play its function. With its inhibition constant of $165 \mathrm{pM}$ against BoNT/A catalytic chain, this biligand is the most potent in vitro inhibitor to date. Furthermore, this inhibitor displayed the ability to protect and rescue BoNT intoxication in a human neuron model.

\section{Protein production \& analytics}

One of the known drawbacks of KTGS that impairs its wider use is surely the need for near-stoichiometric amounts of protein. Access to large homogeneous samples of protein indeed limits access of most drug discovery programs to KTGS as, in comparison, classical screening techniques would allow the screening of tens of thousands of compounds with similar amount of protein. To circumvent this, detection systems that are both selective and highly sensitive have been developed.

The use of LC-MS-TOF [18] has already allowed better signal/noise ratios. Also, some teams have produced specific mutants of the protein of interest to enhance the catalytic turnover [42]. Though this requires specific protein production and engineering, this is of high interest for the future of KTGS.

\section{Looking even further}

As the Thermal Shift Assay methodology has been adapted to the cell context to measure target occupation in a living cell or organism [78], we could dream in the next decade of cell-based KTGS, where the reagents would be able to come in contact with the target in its most relevant context. To detect the formation of the product, high-resolution/high sensitivity mass spectrometers (possibly performed on isotopically labeled reagents to increase specificity) could be combined with the measurement of the pharmacological response to target inhibition by the product in the cell.

Finally, to increase the power of KTGS, it is necessary to expand reagent diversity and number. As the amount of reagents needed for an experiment are very low, the community of researchers interested in KTGS could share the cost of assembling a large, open-source collection of azides and alkynes. In the coming years, the benefit of this unique synthesis approach could really be maximized and obtained at the 'marginal cost.'

\section{Financial \& competing interests disclosure}

The authors have no relevant affiliations or financial involvement with any organization or entity with a financial interest in or financial conflict with the subject matter or materials discussed in the manuscript. This includes employment, consultancies, honoraria, stock ownership or options, expert testimony, grants or patents received or pending, or royalties.

No writing assistance was utilized in the production of this manuscript.

\section{Open access}

This work is licensed under the Attribution-NonCommercial-NoDerivatives 4.0 Unported License. To view a copy of this license, visit http://creativecommons.org/licenses/bync-nd/4.0/

\section{References}

Papers of special note have been highlighted as:

- of interest; $\bullet \bullet$ of considerable interest

1 Schmidt MF, Rademann JR. Dynamic template-assisted strategies in fragment-based drug discovery. Trends Biotechnol. 27(9), 512 (2009).

2 Hochgürtel M, Kroth H, Piecha D et al. Target-induced formation of neuraminidase inhibitors from in vitro virtual combinatorial libraries. Proc. Natl Acad. Sci. USA 99(6), 3382-3387 (2002).

$3 \mathrm{Hu}$ X, Manetsch R. Kinetic target-guided synthesis. Chem. Soc. Rev. 39(4), 1316-1324 (2010).

- A recent review in the field.
4 Mamidyala SK, Finn MG. In situ click chemistry: probing the binding landscapes of biological molecules. Chem. Soc. Rev. 39(4), 1252-1261 (2010).

5 Lewis WG, Green LG, Grynszpan F et al. Click chemistry in situ: acetylcholinesterase as a reaction vessel for the selective assembly of a femtomolar inhibitor from an array of building blocks. Angew. Chem., Int. Ed. Engl. 41(6), 1053 (2002).

-• The first example of in situ click chemistry using Huisgen reaction and the first example of AChE-templated reaction.

6 Rostovtsev VV, Green LG, Fokin VV, Sharpless KB. A stepwise Huisgen cycloaddition process: copper(I)-catalyzed regioselective "ligation" of azides and terminal alkynes. Angew. Chem., Int. Ed. Engl. 41(14), 2596-2599 (2002). 
7 Tornoe CW, Christensen C, Meldal M. Peptidotriazoles on solid phase: [1,2,3]-triazoles by regiospecific copper(I)catalyzed 1,3-dipolar cycloadditions of terminal alkynes to azides. J. Org. Chem. 67(9), 3057-3064 (2002).

8 Jewetta JC, Bertozzi CR. Cu-free click cycloaddition reactions in chemical biology. Chem. Soc. Rev. 39(4), 1272-1279 (2010).

9 Duval R, Kolb S, Braud E, Genest D, Garbay C. Rapid discovery of triazolobenzylidene-thiazolopyrimidines (TBTP) as CDC25 phosphatase inhibitors by parallel click chemistry and in situ screening. J. Comb. Chem. 11(6), 947-950 (2009).

10 Duval R, Kolb S, Braud E, Genest D, Garbay C. Rapid discovery of triazolobenzylidene-thiazolopyrimidines (TBTP) as CDC25 phosphatase inhibitors by parallel click chemistry and in situ screening. J. Comb. Chem. 11(6), 947-950 (2009).

11 Díaz L, Casas J, Bujons J, Llebaria A, Delgado A. New glucocerebrosidase inhibitors by exploration of chemical diversity of n-substituted aminocyclitols using click chemistry and in situ screening. J. Med. Chem. 54(7), 2069-2079 (2011).

12 Tsai J, Lee JT, Wang W et al. Discovery of a selective inhibitor of oncogenic B-Raf kinase with potent antimelanoma activity. Proc. Natl Acad. Sci. USA 105(8), 3041-3046 (2008).

13 Murray CW, Verdonk ML, Rees DC. Experiences in fragment-based drug discovery. Trends Pharmacol. Sci. 33(5), 224-232 (2012).

14 Bienstock RJ. Overview: Fragment-Based Drug Design in Library Design, Search Methods, and Applications of FragmentBased Drug Design. Bienstock R (Ed.). ACS Symposium Series; American Chemical Society, Washington, DC, USA (2011).

15 Grimster NP, Stump B, Fotsing JR et al. Generation of candidate ligands for nicotinic acetylcholine receptors via in situ click chemistry with a soluble acetylcholine binding protein template. J. Am. Chem. Soc. 134(15), 6732-6740 (2012).

16 Willand N, Desroses M, Toto P et al. Exploring drug target flexibility using in situ click chemistry: application to a mycobacterial transcriptional regulator. ACS Chem. Biol. 5(11), 1007-1013 (2010).

- A nice example of the use of multicomponent in situ click chemistry to unravel conformations of a receptor.

17 Mocharla VP, Colasson B, Lee LV et al. In situ click chemistry: enzyme-generated inhibitors of carbonic anhydrase II. Angew. Chem., Int. Ed. Engl. 44(1), 116 (2005).

18 Whiting M, Muldoon J, Lin Y-C et al. Inhibitors of HIV-1 protease by using in situ click chemistry. Angew. Chem., Int Ed. Engl. 45(9), 1435-1439 (2006).

19 Deprez-Poulain R, Nathalie H, Bosc D et al. Catalytic site inhibition of insulin-degrading enzyme by a small molecule induces glucose intolerance in mice. Nat. Commun. 6, 8250 (2015).

-. The first example of orthogonal multicomponent kinetic target-guided synthesis (KTGS) and the first use of a templated-ligand in vivo for pharmacokinetics and pharmacodynamics explorations.
20 Hirose T, Sunazuka T, Sugawara A et al. Chitinase inhibitors: extraction of the active framework from natural argifin and use of in situ click chemistry. J. Antibiot. 62(5), 277 (2009).

21 Asaba T, Suzuki T, Ueda R, Tsumoto H, Nakagawa H, Miyata N. Inhibition of human sirtuins by in situ generation of an acetylated lysine-ADP-ribose conjugate. J. Am. Chem. Soc. 131(20), 6989-6996 (2009).

22 Peruzzotti C, Borrelli S, Ventura M et al. Probing the binding site of Abl tyrosine kinase using in situ click chemistry. ACS Med. Chem. Lett. 4(2), 274-277 (2013).

- Proof-of-concept of the use of KTGS for kinase-templated reactions.

23 Gelin M, Poncet-Montange G, Assairi L et al. Screening and in situ synthesis using crystals of a NAD kinase lead to a potent antistaphylococcal compound. Structure 20(6), 1107-1117 (2012).

24 Meyerkord CL, Fu H, Fry D. Targeting protein-protein interactions for drug discovery. In: Protein-Protein Interactions. Springer, NY, USA, 93-106 (2015).

25 Mullard A. Protein-protein interaction inhibitors get into the groove. Nat. Rev. Drug Discov. 11(3), 173-175 (2012).

26 Hu X, Sun J, Wang H, Manetsch R. Bcl-X-L-templated assembly of its own protein-protein interaction modulator from fragments decorated with thio acids and sulfonyl azides. J. Am. Chem. Soc. 130 (42), 13820-13821 (2008).

-• The first example of KTGS used to investigate protein-protein interactions and sulfo-click chemistry.

27 Kulkarni SS, Hu X, Doi K, Wang H-G, Manetsch R. Screening of protein-protein interaction modulators via sulfo-click kinetic target-guided synthesis. ACS Chem. Biol. 6, 724-732 (2011)

28 Maki T, Kawamura A, Kato N, Ohkanda J. Chemical ligation of epoxide-containing fusicoccins and peptide fragments guided by 14-3-3 protein. Mol. BioSyst. 9(5), 940-943 (2013).

29 Silman I, Sussman JL. Acetylcholinesterase: "classical" and "non-classical" functions and pharmacology. Curr. Opin. Pharmacol. 5(3), 293-302 (2005).

30 Nikas SP, Sharma R, Paronis CA et al. Probing the carboxyester side chain in controlled deactivation $(-)-\delta(8)-$ tetrahydrocannabinols. J. Med. Chem. 58(2), 665-681 (2015).

31 Krasiński A, Radić Z, Manetsch R et al. In situ selection of lead compounds by click chemistry: target-guided optimization of acetylcholinesterase inhibitors. J. Am. Chem. Soc. 127(18), 6686-6692 (2005).

- One of the first examples of multicomponent KTGS with small sets of reagents.

32 Manetsch R, Krasiński A, Radić Z et al. In situ click chemistry: enzyme inhibitors made to their own specifications. J. Am. Chem. Soc. 126(40), 12809-12818 (2004).

33 Oueis E, Santoni G, Ronco C et al. Reaction site-driven regioselective synthesis of AChE inhibitors. Org. Biomol. Chem. 12(1), 156 (2014). 
34 Oueis E, Nachon F, Sabot C, Renard P-Y. First enzymatic hydrolysis/thio-Michael addition cascade route to synthesis of AChE inhibitors. Chem. Commun. 50(16), 2043 (2014). Jones Ck, Byun N, Bubser M. Muscarinic and nicotinic acetylcholine receptor agonists and allosteric modulators for the treatment of schizophrenia. Neuropsychopharmacology 37(1), 16-42 (2012).

36 Russo P, Del Bufalo A, Frustaci A, Fini M, Cesario A. Beyond acetylcholinesterase inhibitors for treating Alzheimer's disease: alpha7-nAChR agonists in human clinical trials. Curr. Pharm. Des. 20(38), 6014-6021 (2014).

37 Frost SC, Mckenna R, Scozzafava A, Supuran C. Glaucoma and the applications of carbonic anhydrase inhibitors. In: Carbonic Anhydrase: Mechanism, Regulation, Links to Disease, and Industrial Applications. Springer, The Netherlands, 349-359 (2014).

38 Carta F, Supuran CT. Diuretics with carbonic anhydrase inhibitory action: a patent and literature review (2005 -2013). Expert Opin. Ther. Pat. 23(6), 681-691 (2014).

39 Nguyen R, Huc I. Using an enzyme's active site to template inhibitors. Angew. Chem., Int. Ed. Engl. 40(9), 1774 (2001).

-. First example of KTGS on carbonic anhydrase.

40 Mocharla VP, Walsh JC, Padgett HC et al. From in situ to in vivo: an in situ click-chemistry-derived carbonic anhydrase II imaging agent for positron emission tomography. ChemMedChem 8(1), 43-48 (2013).

- The use of KTGS to discover new compounds precursors of PET-imaging probes.

41 SIEMENS MEDICAL SOLUTIONS INC.: WO2006116736A2 (2006).

42 Willand N, Dirié B, Carette X et al. Synthetic EthR inhibitors boost anti-tuberculous activity of ethionamide. Nat. Med. 15(5), 537-544 (2009).

43 Tieu W, Soares Da Costa TP, Yap MY et al. Optimising in situ click chemistry: the screening and identification of biotin protein ligase inhibitors. Chem. Sci. 4(9), 3533-3537 (2013).

- A report on how proteins can be engineered to enhance the KTGS yield.

44 Bodur C, Basaga H. Bcl-2 inhibitors: emerging drugs in cancer therapy. Curr. Med. Chem. 19(12), 1804-1820 (2015).

$45 \mathrm{Hu}$ X, Manetsch R. Kinetic target-guided synthesis. Chem. Soc. Rev. 39(4), 1316-1324 (2010).

46 Oueis E, Sabot C, Renard PY. New insights into the kinetic target-guided synthesis of protein ligands Chem. Commun. 51, 12158-12169 (2015).

47 Kolb HC, Finn MG, Sharpless KB. Click chemistry: diverse chemical function from a few good reactions. Angew. Chem., Int. Ed. Engl. 40 (11), 2004-2021 (2001).

48 Mock WL, Irra TA, Wepsiec JP, Manimaran TL. Cycloaddition induced by cucurbituril - a case of Pauling principle catalysis. J. Org. Chem. 48 (20), 3619-3620 (1983).

49 Mock WL, Irra TA, Wepsiec JP, Adhya M. Catalysis by curcurbituril - the significance of bound-substrate destabilization for induced triazole formation. J. Org. Chem. 54 (22), 5302-5308 (1989).

50 Michael A. Ueber die Einwirkung von Diazobenzolimid auf Acetylendicarbonsäure methylester, J. Prakt. Chem. 48, 94-95 (1893).

51 Shangguan N, Katukojvala S, Greenburg R, Williams LJ. The reaction of thio acids with azides: a new mechanism and new synthetic applications. J. Am. Chem. Soc. 125 (26), 7754-7755 (2003).

52 Kolakowski RV, Shangguan N, Sauers RR, Williams LJ. Mechanism of thio acid/azide amidation. J. Am. Chem. Soc. 128 (17), 5695-5702 (2006).

53 Chase JFA Tubbs PK. Conditions for self-catalysed inactivation of carnitine acetyltransferase - a novel form of enzyme inhibition. Biochem. J. 111, 225-235 (1969).

54 Shi B, Stevenson R, Campopiano DJ, Greaney MF. Discovery of glutathione $S$-transferase inhibitors using dynamic combinatorial chemistry. J. Am. Chem. Soc. 128(26), 8459-8467 (2006).

55 Hirose T, Maita N, Gouda $\mathrm{H}$ et al. Observation of the controlled assembly of preclick components in the in situ click chemistry generation of a chitinase inhibitor. Proc. Natl Acad. Sci. USA 110 (40), 15892-15897 (2013).

-• A nice example of the observation by $x$-ray diffraction of click reagents in the protein.

56 Rostovtsev VV, Green LG, Fokin VV, Sharpless KB. A stepwise huisgen cycloaddition process: copper(I)-catalyzed regioselective "ligation" of azides and terminal alkynes. Angew. Chem., Int. Ed. Engl. 41(14), 2596-2599 (2002).

57 Tornoe CW, Christensen C, Meldal M. Peptidotriazoles on solid phase: [1,2,3]-triazoles by regiospecific copper(I)catalyzed 1,3-dipolar cycloadditions of terminal alkynes to azides. J. Org. Chem. 67(9), 3057-3064 (2002).

58 Zhang L, Chen X, Xue P et al. Ruthenium-catalyzed cycloaddition of alkynes and organic azides. J. Am. Chem. Soc. 127(46), 15998-15999 (2005).

59 Boren BC, Narayan S, Rasmussen LK et al. Rutheniumcatalyzed azide-alkyne cycloaddition: scope and mechanism. J. Am. Chem. Soc. 130 (28), 8923-8930 (2008).

60 Deprez B, Williard X, Bourel L, Coste H, Hyafil F, Tartar A. Orthogonal combinatorial chemical libraries. J. Am. Chem. Soc. 117(19), 5405 (1995).

- The article at the origin of orthogonal libraries.

61 Erlanson DA, Mcdowell RS, O’Brien T. Fragment-based drug discovery. J. Med. Chem. 47(14), 3463 (2004).

62 Chase JFA, Tubbs PK. Conditions for self-catalysed inactivation of carnitine acetyltransferase - a novel form of enzyme inhibition. Biochem. J. 111, 225-235 (1969).

63 Bourne Y, Kolb HC, Radic Z, Sharpless KB, Taylor P, Marchot P. Freeze-frame inhibitor captures acetylcholinesterase in a unique conformation. Proc. Natl Acad. Sci. USA 101(6), 1449-1454 (2004).

64 Mugnaini C, Nocerino S, Pedani V et al. Investigations on the 4-quinolone-3-carboxylic acid motif part 5: modulation of the physicochemical profile of a set of potent and selective 
cannabinoid-2 receptor ligands through a bioisosteric approach. ChemMedChem 7(5), 920-934 (2012).

$65 \mathrm{Li} \mathrm{H}$, Aneja R, Chaiken I. Click chemistry in peptide-based drug design. Molecules 18(8), 9797-9817 (2013).

66 Soares Da Costa TP, Tieu W, Yap MY et al. Selective inhibition of biotin protein ligase from Staphylococcus aureus. J. Biol. Chem. 287(21), 17823-17832 (2012).

67 Lau JK-C, Wong CHS, Ng PS, Siu FM, Ma NL, Tsang CW. Absolute potassium cation affinities (PCAs) in the gas phase. Chem. Eur. J. 9(14), 3383 (2003).

68 Brown RD, Coller BAW, Kent JE. 1, 2, 5-, 1, 3,4- and 1,2,4-oxadiazoles: a theoretical study of electric dipole moments. Theor. Chim. Acta 10, 435-446 (1968).

69 Namelikonda NK, Manetsch R. Sulfo-click reaction via in situ generated thioacids and its application in kinetic target-guided synthesis. Chem. Commun. (Camb.) 48(10), 1526-1528 (2012).

70 Weber L. In vitro combinatorial chemistry to create drugs, Drug Discov. Today 1(3), 261-267 (2004).

71 Malaquin S, Jida M, Courtin J et al. Water-based conditions for the microscale parallel synthesis of bicyclic lactams. Tetrahedron Lett. 54(6), 562-567 (2013).

72 Kamber DN, Liang Y, Blizzard RJ et al. 1,2,4-triazines are versatile bioorthogonal reagents. J. Am. Chem. Soc. 137(26), 8388-8391 (2015).
73 Wang X, Huang B, Liu X, Zhan P. Discovery of bioactive molecules from CuAAC click-chemistry-based combinatorial libraries. Drug Discov. Today S1359-S6446(15), 320-327 (2015).

74 Agnew HD, Rohde RD, Millward SW et al. Iterative in situ click chemistry creates antibody-like protein-capture agents. Angew. Chem., Int. Ed. Engl. 48(27), 4944-4948 (2009).

75 Nag A, Das S, Yu MB, Deyle KM, Millward SW, Heath JR. A chemical epitope-targeting strategy for protein capture agents: the serine 474 epitope of the kinase Akt2. Angew. Chem., Int. Ed. Engl. 52(52), 13975-13979 (2013)

76 Deyle KM, Farrow B, Qiao Hee Y et al. A protein-targeting strategy used to develop a selective inhibitor of the $E 17 K$ point mutation in the PH domain of Akt1. Nat. Chem. 7(5), 455-462 (2015).

77 Farrow B, Wong M, Malette J et al. Epitope targeting of tertiary protein structure enables target-guided synthesis of a potent in-cell inhibitor of botulinum neurotoxin. Angew. Chem., Int. Ed. Engl. 54(24), 7114-7119 (2015).

78 Martinez-Molina D, Jafari R, Ignatushchenko M et al. Monitoring drug target engagement in cells and tissues using the cellular thermal shift assay. Science 341(6141), 84-87 (2013). 